\title{
Mission impossible completed: unlocking the nomenclature of the largest and most complicated subgenus of Cortinarius, Telamonia
}

\author{
Kare Liimatainen $^{1}$ (D) Tuula Niskanen ${ }^{1,2} \mathbb{D} \cdot$ Bálint Dima $^{3}$ (D) Joseph F. Ammirati ${ }^{4}$ - Paul M. Kirk ${ }^{1}$ (D) Ilkka Kytövuori ${ }^{2}$
}

Received: 9 May 2020 / Accepted: 6 August 2020 / Published online: 8 September 2020

(c) The Author(s) 2020

\begin{abstract}
So far approximately 144,000 species of fungi have been named but sequences of the majority of them do not exist in the public databases. Therefore, the quality and coverage of public barcode databases is a bottleneck that hinders the study of fungi. Cortinarius is the largest genus of Agaricales with thousands of species world-wide. The most diverse subgenus in Cortinarius is Telamonia and its species have been considered one of the most taxonomically challenging in the Agaricales. Its high diversity combined with convergent, similar appearing taxa have earned it a reputation of being an impossible group to study. In this study a total of 746 specimens, including 482 type specimens representing 184 species were sequenced. Also, a significant number of old types were successfully sequenced, 105 type specimens were over 50 years old and 18 type specimens over 100 years old. Altogether, 20 epi- or neotypes are proposed for recently commonly used older names. Our study doubles the number of reliable DNA-barcodes of species of $C$. subgenus Telamonia in the public sequence databases. This is also the first extensive phylogenetic study of the subgenus. A majority of the sections and species are shown in a phylogenetic context for the first time. Our study shows that nomenclatural problems, even in difficult groups like $C$. subgenus Telamonia, can be solved and consequently identification of species based on ITS barcodes becomes an easy task even for non-experts of the genus.
\end{abstract}

Keywords ITS $\cdot$ Type study $\cdot$ Barcode $\cdot$ Neotype $\cdot$ Phylogeny $\cdot$ Section

\section{Introduction}

Electronic supplementary material The online version of this article (https://doi.org/10.1007/s13225-020-00459-1) contains supplementary material, which is available to authorized users.

Kare Liimatainen

k.liimatainen@kew.org

Tuula Niskanen

tuula.niskanen@cortinarius.fi

Bálint Dima

cortinarius1@gmail.com

Joseph F. Ammirati

cort@u.washington.edu

Paul M. Kirk

p.kirk@kew.org

Ilkka Kytövuori

ilkka.kytovuori@gmail.com
So far approximately 144,000 species of fungi have been named (Willis 2018) but sequences of the majority of them do not exist in the GenBank or UNITE. Moreover, only a small percentage of the names in the GenBank, about 4800

1 Jodrell Laboratory, Royal Botanic Gardens, Kew, Surrey TW9 3AB, UK

2 Botanical Museum, University of Helsinki, P.O. Box 7, 00014 Helsinki, Finland

3 Department of Plant Anatomy, Institute of Biology, Eötvös Loránd University, Pázmány Péter sétány 1/C, 1117 Budapest, Hungary

4 Department of Biology, University of Washington, Box 351800, Seattle, WA 98195-1800, USA 
species, are based on sequences from type materials or other reliable sources (Schoch et al. 2014).

Currently species identification of fungi in academic studies is almost solely based on nrDNA ITS barcodes (Lindahl et al. 2013). Thus, those collections with taxonomically correct names that are not in any public sequence repositories are basically omitted in academic research. Therefore, the quality and coverage of public barcode databases is a bottleneck that hinders the study of fungi (Schoch et al. 2014). Depositing the ITS sequences in public repositories like GenBank does not automatically make them useful for identification. Two excellent platforms for delivering sequencebased identification information for the end-users include RefSeq under GenBank (Schoch et al. 2014) and UNITE (Kõljalg et al. 2013). However, in both cases an extra step by an expert, in addition to the normal sequence submission, is required, but unfortunately often is left undone, making part of the already existing information unusable.

Cortinarius (Pers.) Gray is the largest genus of Agaricales with thousands of species world-wide (Kirk et al. 2008). They are important ectomycorrhizal fungi and often discovered in ecological studies. Only three large studies of type specimens based on ITS sequence data in Cortinarius have been made so far. Two of them are from $C$. subgen. Phlegmacium (Fr.) Trog: Liimatainen et al. (2014) includes over 230 sequences of type specimens representing over 150 species and Frøslev et al. (2007) has over 50 sequences of 79 species. The third one is from $C$. subgen. Telamonia (Fr.) Trog and includes over 60 sequences of 33 species (Liimatainen et al. 2017).

The most species-rich subgenus in Cortinarius is Telamonia. Members of this subgenus mainly occur in the Northern Hemisphere (Garnica et al. 2005, Soop et al. 2019) and are especially dominant in coniferous forests. Six relatively large phylogenetic studies of Cortinarius have been published so far (Garnica et al. 2005, 2016; Harrower et al. 2011; Peintner et al. 2004; Soop et al. 2019; Stensrud et al. 2014). The number of species of Telamonia included in these studies varies from 8 to 70 , and the phylogenies have been mainly based on the analysis of ITS and LSU regions, with some also including sequences of $r p b 1$ or $r p b 2$ regions. These studies have shown that the majority of the traditionally morphologically delimited species of Telamonia (Bidaud et al. 2017; Brandrud et al. 2012; Moser 1983; Niskanen et al. 2012) form a monophyletic group. However, sections or subgenera that have been shown not to belong to Telamonia include Anomali Konrad \& Maubl., Balaustini Moënne-Locc. \& Reumaux, Camphorati Liimat., Niskanen \& Ammirati, Fulvescentes Melot, Illumini Liimat., Niskanen \& Kytöv., Obtusi Melot, Renidentes Moënne-Locc. \& Reumaux, and Rigentes Melot. Morphological characters that define the species of Telamonia are basidiomata with dry pileus and dry stipe and mainly brownish colours, with the exception of some whitish, bluish-purple or orange-red species. No larger phylogenetic analysis on the infrasubgeneric relationships have been done so far, but studies on specific sections including sequences from type specimens have been published: Armillati M.M. Moser (Niskanen et al. 2011), Bovini M.M. Moser (Niskanen et al. 2013), Brunnei Melot (Niskanen et al. 2009), Colymbadini Melot/Cinnabarini Melot/Uracei Melot (Ammirati et al. 2017; Dima et al. 2014), Disjungendi Kytöv., Liimat., Niskanen \& Ammirati (Liimatainen et al. 2015), Hydrocybe (Fr. ex Rabenh.) Gillot \& Lucand (Suárez-Santiago et al. 2009) and Saturnini Moënne-Locc. \& Reumaux/Bicolores (M.M. Moser) Melot (Liimatainen et al. 2017).

The nuclear ribosomal internal transcribed spacer (ITS), which has been proposed as the universal barcode marker for fungi (Schoch et al. 2012), is also the main locus used in the species level taxonomy of Cortinarius. The treshold value for barcoding Cortinarius species has been proposed to be $99 \%$ (Garnica et al. 2016). However, there already is evidence that a few morphologically distinct Telamonia species only have 1 base difference ( $99.8 \%$ similarity) in the ITS region, e.g. C. laniger Fr./C. solis-occaus Melot (Niskanen et al. 2012) and $C$. paragaudis Fr./C. pinigaudis Niskanen, Kytöv. \& Liimat. (Niskanen et al. 2011) and in the case of $C$. confirmatus Rob. Henry the intraspecific variation is $>1 \%$, although the species has a wide morphological and ecological range and based on ITS sequences there are 3 supported subclades which might be separate taxa (Liimatainen et al. 2017).

In this study our aim was to provide a revision of Cortinarius, subgen. Telamonia as well as an extensive ITS database for the identification of the species. Almost all type specimens of the species described in the subgenus were studied and an epi- or neotype is proposed for all recent frequently used older names when possible. In addition, a phylogenetic tree is produced as a framework for the infrasubgeneric classification of the species; including many that are included in a phylogenetic analysis for the first time.

\section{Materials and methods}

\section{Taxon sampling}

The type specimens of Telamonia species published over many years by Ammirati, D. Antonini, M. Antonini, Bergeron, Bidaud, Bohus, Bouteville, Bresadola, Carteret, Chevassut, Consiglio, Daniele, Eyssartier, A. Favre, J. Favre, Fellner, Ferville, Fillion, Henry, Hesler, Høiland, Hongo, Karsten, Kauffman, Kühner, Landa, Lindström, Matheny, McKnight, Moser, Moënne-Loccoz, Murril, Nespiak, Orton, Pearson, Peck, Ramm, Reumaux, Sasia, Seidl, Smith, Schwöbel, Soop, Svrček, Velenovský, and Vialard were sampled as well as all the Telamonia collections published and illustrated in Brandrud et al. (1989, 1992, 1994, 1998). A total of 482 types are included here. An additional 
183 previously published sequences of Telamonia types were added to our dataset for the best overview of current available data.

We aimed to have at least two sequences per species in our study. Therefore, some additional sequences, either our own unpublished ones or from databases GenBank and UNITE, were included. Information on the sequences of type specimens is available in Supplementary Table 1 and information on other sequences included in the phylogenetic analysis is available in Supplementary Table 2. Fungarium acronyms follow Index Herbariorum (Thiers 2013).

\section{Species concept}

Based on criteria mentioned in the introduction we have used $1 \%$ (5 differences) as a cut-off value for species. When type sequences differ in at least 5 sites from one another we have treated them as different species. We are not claiming that all the variation below $1 \%$ is automatically intraspecific. Separating species below the $1 \%$ cut-off value, however, does require careful study. Therefore, we have added 'aff.' prefix to the Latin name in cases where there are 3 to 4 differences to another type sequences. With 2 differences we have used the $s$. lato notation in the Fig. 1 and Supplementary Tables 1 and 2. Using this approach indicates places where determining taxonomic synonyms might be problematic and require further study. Also, when macroscopic, microscopic and/or ecology data differ considerably although the ITS sequences are the same, we have not placed the taxa in synonomy. Furthermore, in cases where a species complex has previously been shown to include several species supported by morphology and small, but constant barcode gaps, we have avoided making synonymys.

One cannot emphasize enough that using a small cut-off value requires good quality sequences. In this study all the specimens have been sequenced from both directions and the chromatograms of the sequences were checked and edited manually before any preliminary analyses. When small, less than ten base or indel changes and/or odd differences are found between sister species or within species those differences have been confirmed by combining the relevant chromatograms and checking manually the base sites that differ. Also, base or length polymorphisms sites are not counted as a difference and an indel is counted as one difference despite its length.

\section{Molecular analyses}

DNA was extracted from a few milligrams of dried material (a piece of lamella) with the NucleoSpin Plant kit (Macherey-Nagel, Düren, Germany). The same protocol was used for all materials. Primers ITS 1F and ITS 4 (Gardes and Bruns 1993; White et al. 1990) were used to amplify ITS regions. The same primer pairs were used in direct sequencing. For problematic material the primer combinations ITS
1F/ITS 2 and ITS 3/ITS 4 were also used. PCR amplifications were performed in a $25 \mu \mathrm{l}$ reaction mix with about 70 ng extracted DNA, 1 U Phusion High-Fidelity DNA polymerase and $1 \times$ HF buffer (ThermoFisher), $200 \mathrm{mM}$ of each dNTP and $0.5 \mu \mathrm{M}$ of each primer. The PCR were run on a MBS 0.2 G Thermal Cycler (Thermo Hybaid) with the following settings: denaturation for $30 \mathrm{~s}$ at $98{ }^{\circ} \mathrm{C}$, followed by 35 cycles of denaturation for $10 \mathrm{~s}$ at $98^{\circ} \mathrm{C}$, annealing for $30 \mathrm{~s}$ at $50{ }^{\circ} \mathrm{C}$, and extension for $30 \mathrm{~s}$ at $72{ }^{\circ} \mathrm{C}$. The PCR products were purified using an ExoSAP-IT purification kit (Amersham Biosciences). Sequencing was performed on both strands using a BigDye Terminator v1.1 Sequencing kit (Applied Biosystems). Reactions were performed in $10 \mu \mathrm{l}$ with $1 \mu \mathrm{l}$ of PCR product, $1.3 \mathrm{mM}$ of primer (ITS $1 \mathrm{~F}$ or ITS 4), $1 \mu \mathrm{l} 5 \mathrm{X}$ sequencing buffer, and $1 \mu \mathrm{l}$ of Terminator Ready Reaction Mix. Reactions were run for $1 \mathrm{~min}$ at $96^{\circ} \mathrm{C}$, followed by 30 cycles of $30 \mathrm{~s}$ at $96^{\circ} \mathrm{C}, 15 \mathrm{~s}$ at $50^{\circ} \mathrm{C}$, and $4 \mathrm{~min}$ at $60{ }^{\circ} \mathrm{C}$. Unincorporated dye terminators and primers were removed by Sephadex G-50 DNA Grade Fine (Amersham Biosciences) purification system, and the reactions were analysed by ABI 3730 DNA Analyzer (Applied Biosystems) automatic sequencer. Sequences were assembled and edited with Sequencher 4.1 (Gene Codes, Ann Arbor, Michigan, USA). A total of 755 new ITS sequences were produced for this study. Collections and GenBank sequences used for the phylogenetic analysis are given in Supplementary Tables 1 and 2.

The short ITS sequences of type specimens were excluded from the phylogenetic analysis. To improve the resolution of phylogenetic analyses we included 146 published LSU sequences from GenBank to our dataset. The chosen LSU sequences are from different parts of Telamonia and they were mostly obtained from Garnica et al. (2005), Harrower et al. (2011), and Stensrud et al. (2014). Sequences from section Dermocybe Pers. were selected as an outgroup based on Stensrud et al. (2014). A total of 919 ITS and 146 LSU sequences were aligned separately for both regions using MAFFT 7 (Katoh and Standley 2013) with the G-ING-i algorithm (Katoh et al. 2005). The alignments were then manually improved in SeaView (Galtier et al. 1996). The phylogenetically informative indels in the ITS region were coded as characters following the simple indel coding algorithm (Simmons and Ochoterena 2000) with FastGap 1.2 (Borchsenius 2009). The binary and aligned nucleotide data were concatenated in Mesquite 3.2 (Maddison and Maddison 2017). The alignment is 2008 nucleotides long (including gaps) and is available at TreeBASE under S26824 (http:// www.treebase.org/treebase-web/home.html). A phylogenetic tree was generated from the concatenated dataset using maximum likelihood (ML) analyses with 1000 bootstrap replicates under the GTRGAMMA model for nucleotide partitions (ITS + LSU) and the default setting for binary (indel) data in RAxML 8 (Stamatakis 2014). 
Fig. 1 A phylogram resulting from the RAxML analysis of the concatenated dataset including 919 aligned ITS and 146 LSU sequences and the binary data resulting from the coding of the phylogenetically informative insertions/deletions in the ITS alignment. Bootstrap values greater than $50 \%$ are indicated above branches. The current names of species are in boldface

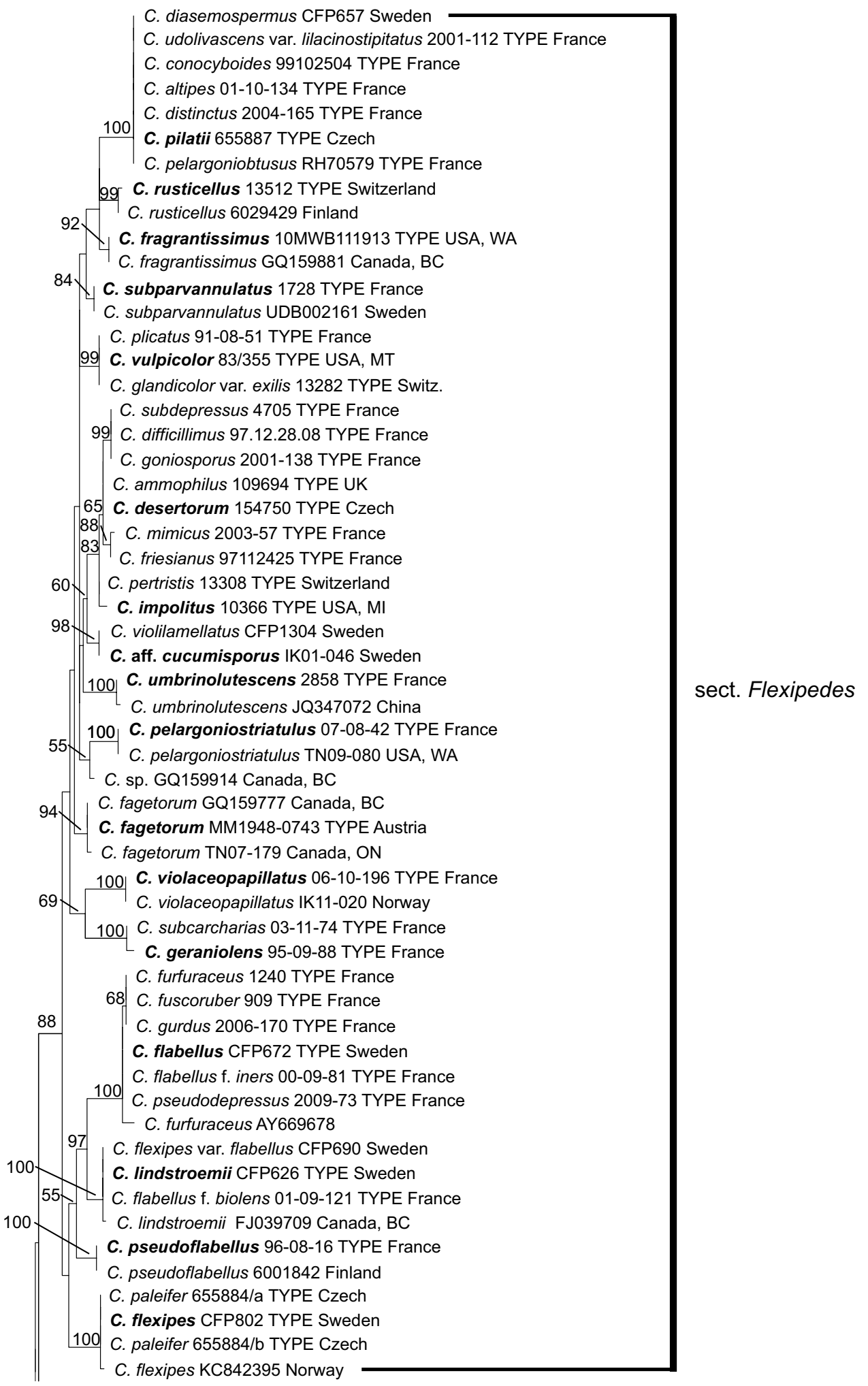


Fig. 1 (continued)

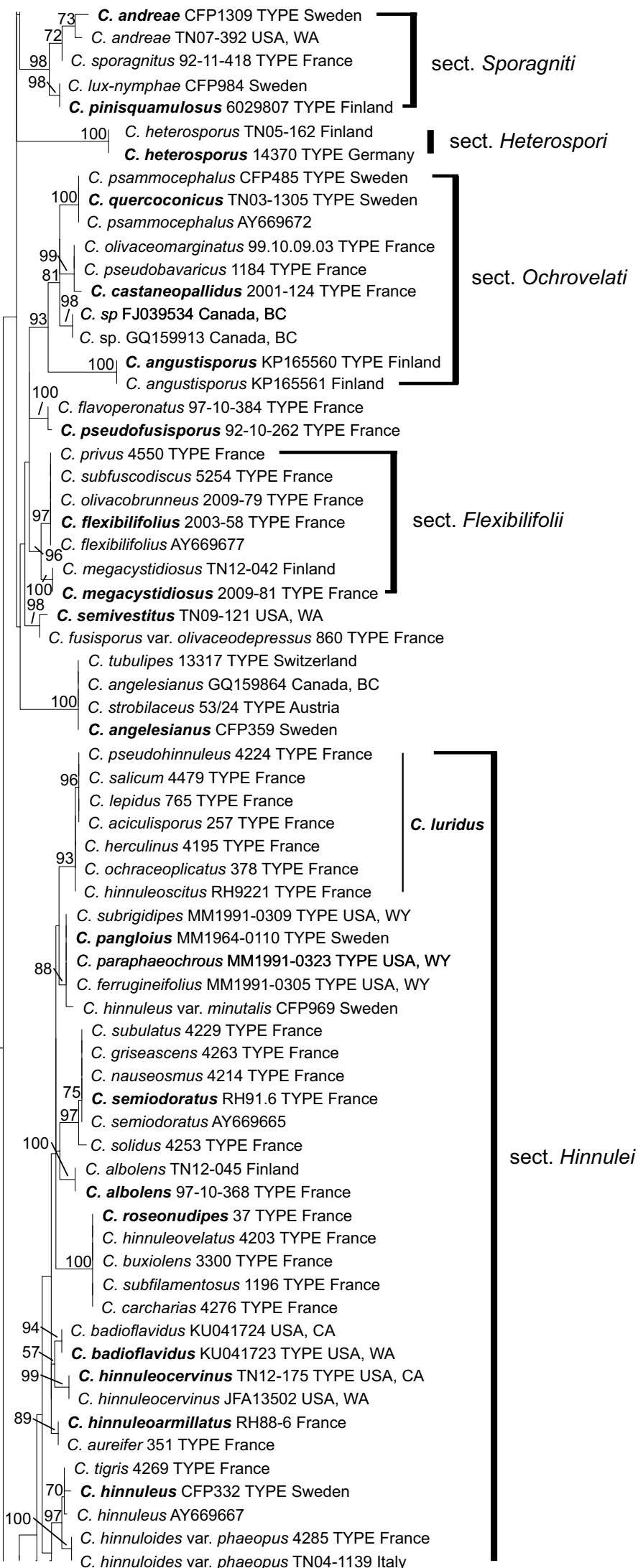


Fig. 1 (continued)

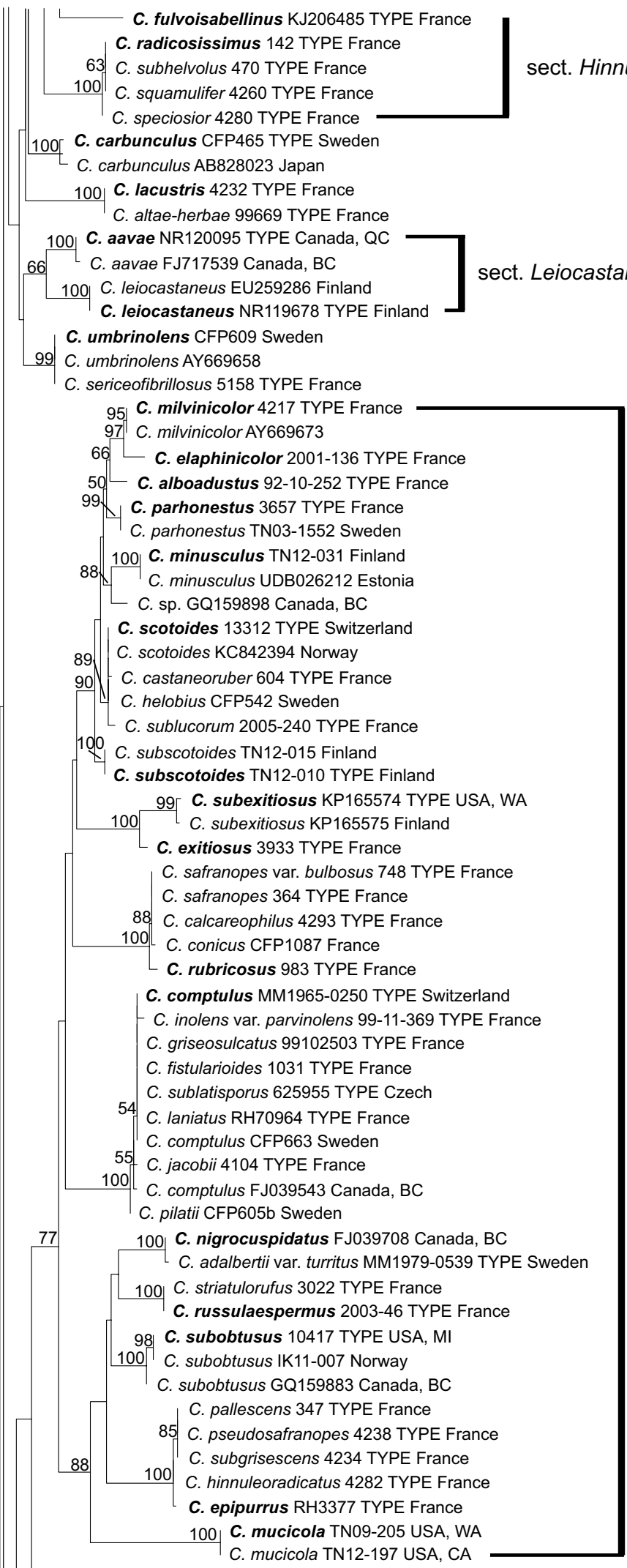

sect. Rubricosi 
Fig. 1 (continued)

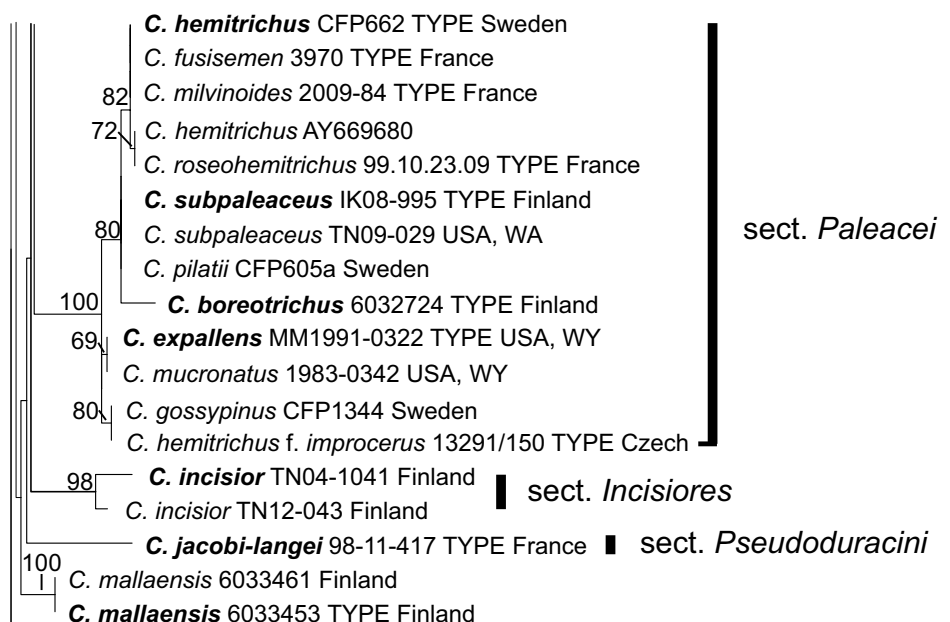

C. saniosus DQ102671 TYPE Sweden

C. bavaricus MM1967-0102 TYPE Germany

C. Iuteolateritius 154749 TYPE Czech

60. C. rufoanuliferus 1983/386 TYPE USA, WY C. saniosus AY 669660

100 C. subaurantiomarginatus 5249 TYPE France

C. saniosus GQ159783 Canada, BC

90 C. aff. gentilissimus TN10-177 Canada, QC

C. aureovelatus DQ102652 TYPE Norway

100 C. aff. gentilissimus IK01-041 Finland

sect. Saniosi

$69100-$ C. cf. gentilissimus JFA11923 Costa Rica

C. cf. gentilissimus JFA12032 Costa Rica

78

96 C. saniosus var. paludophilus 1182 TYPE France

100 C. paludosaniosus TN07-342 TYPE USA, WA

C. paludosaniosus AY669621

100 C. fuscogracilescens TN03-1348 Sweden

96 C. fuscogracilescens 00274011 TYPE France

C. corvinus 1188 TYPE France

C. americanus GQ398244 USA, OR

86. C. americanus TN07-473 USA, WA

C. americanus 10314 TYPE USA, MI

100 C. sphagnicola 3934 TYPE France

C. americanus HE979238 China

sect. Bibuli

100 C. bibulus UDB002795 Estonia

C. bibulus TN05-119 TYPE Finland

100 C. fuscodiscus FJ039555 Canada, BC

70 C. fuscodiscus TN09-069 USA, WA

100 C. sphagnoravus AY669683

C. sphagnoravus CFP746 TYPE Sweden

sect. Ravi

99 C. atripes 436 TYPE France

100 C. griseocarneus 2004-163 TYPE France

69y C. alnetorum CFP339 Sweden

C. griseocarneus AY669695

C. helvelloides AY669684

63 C. helvelloides TN05-002 TYPE Finland

60 C. helvelloides CFP340 Sweden

93. C. helodes FJ039707 Canada, BC

100 C. helodes MM1997-0311 TYPE USA

93. C. lacorum 10370 TYPE USA, MI

C. olivaceofulvus 10384 TYPE USA, MI

sect. Alnicolarum

C. badiovestitus MM1965-0040 TYPE Austria

66 C. erythrinus var. russulisporus 58132 TYPE Hungary

100 C. petroselineus var. radicipes FN429003 TYPE France

93 C. vernus UDB000742 TYPE Sweden

C. sp. FJ039541 Canada, BC

sect. Verni 
Fig. 1 (continued)

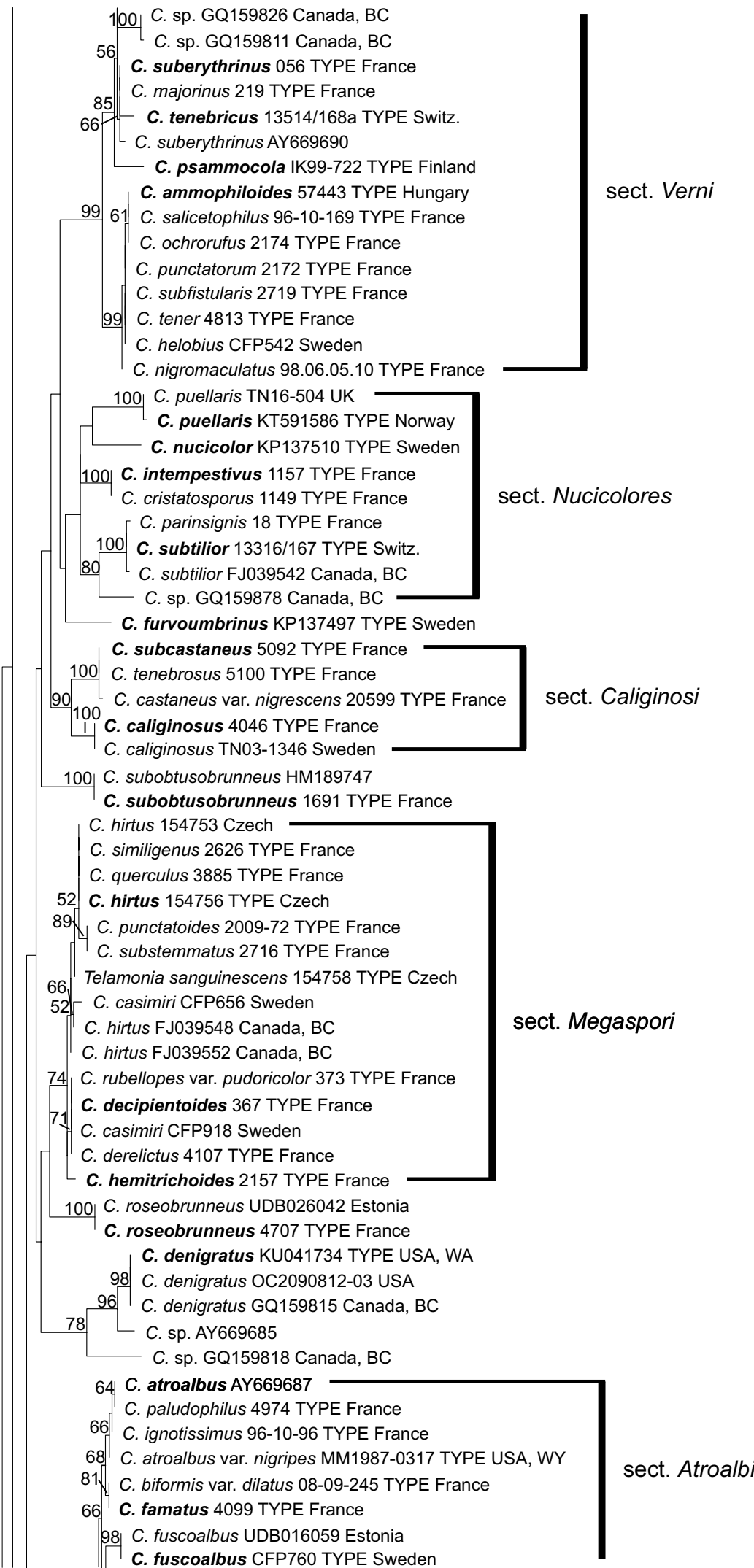


Fig. 1 (continued) 
Fig. 1 (continued)

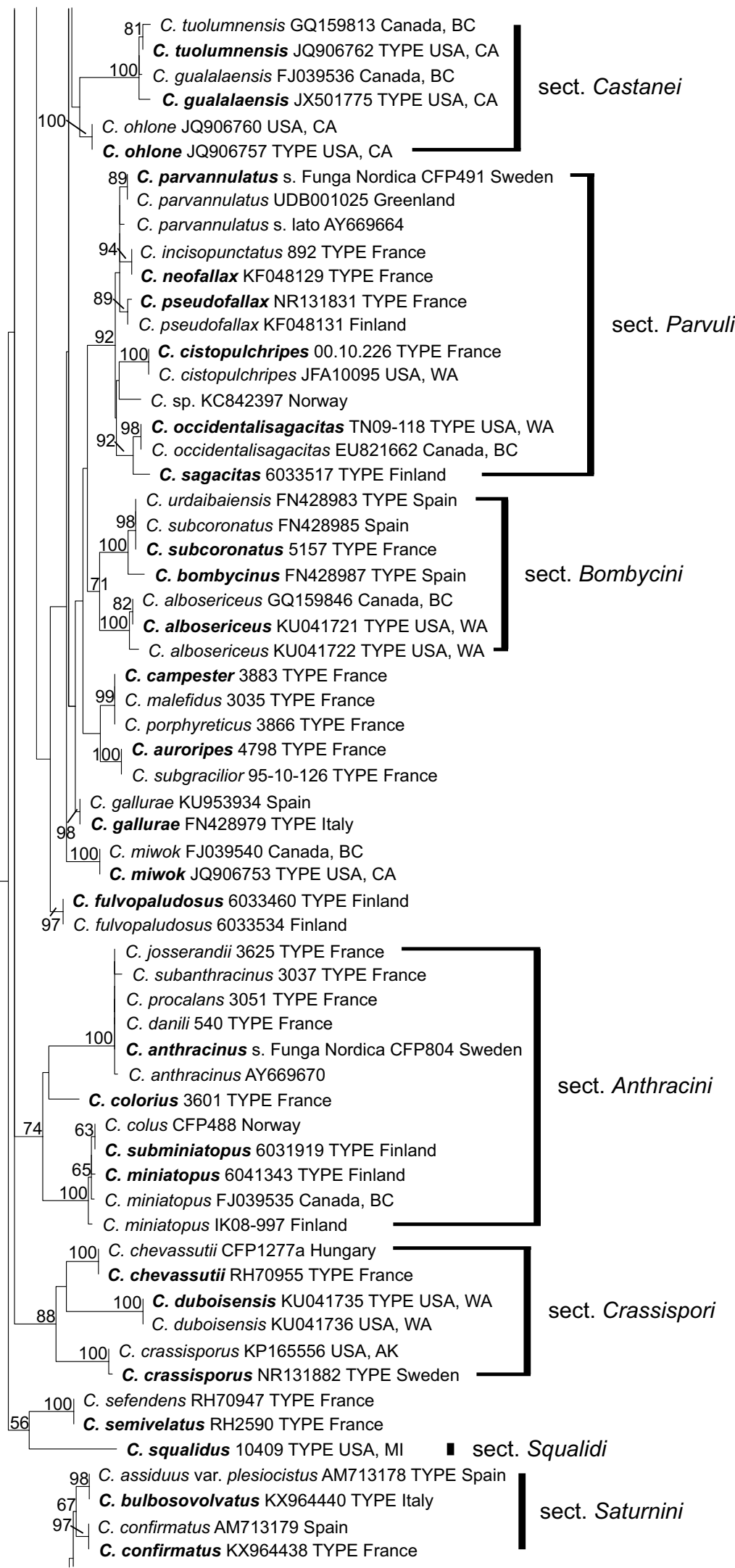


Fig. 1 (continued)

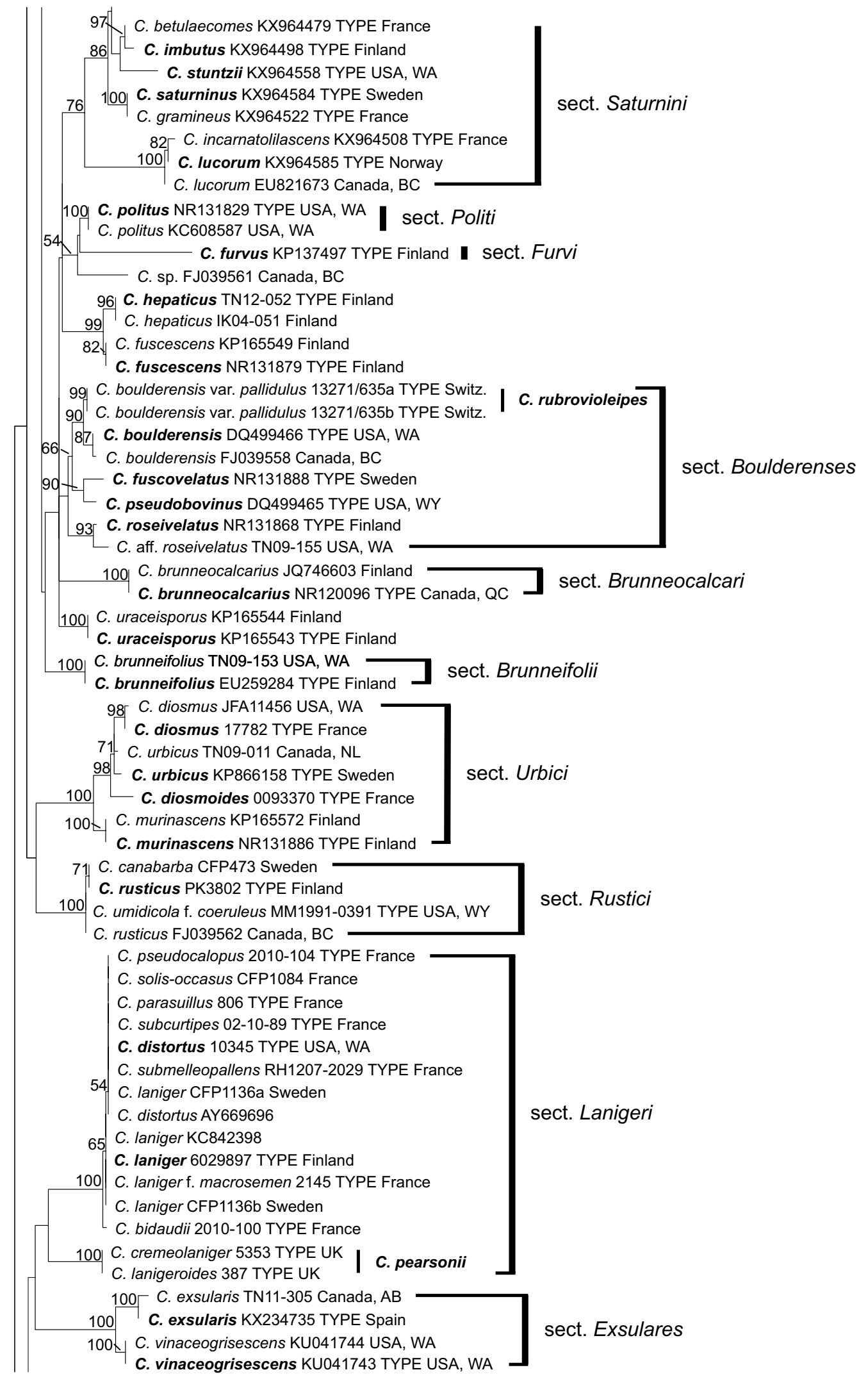


Fig. 1 (continued)

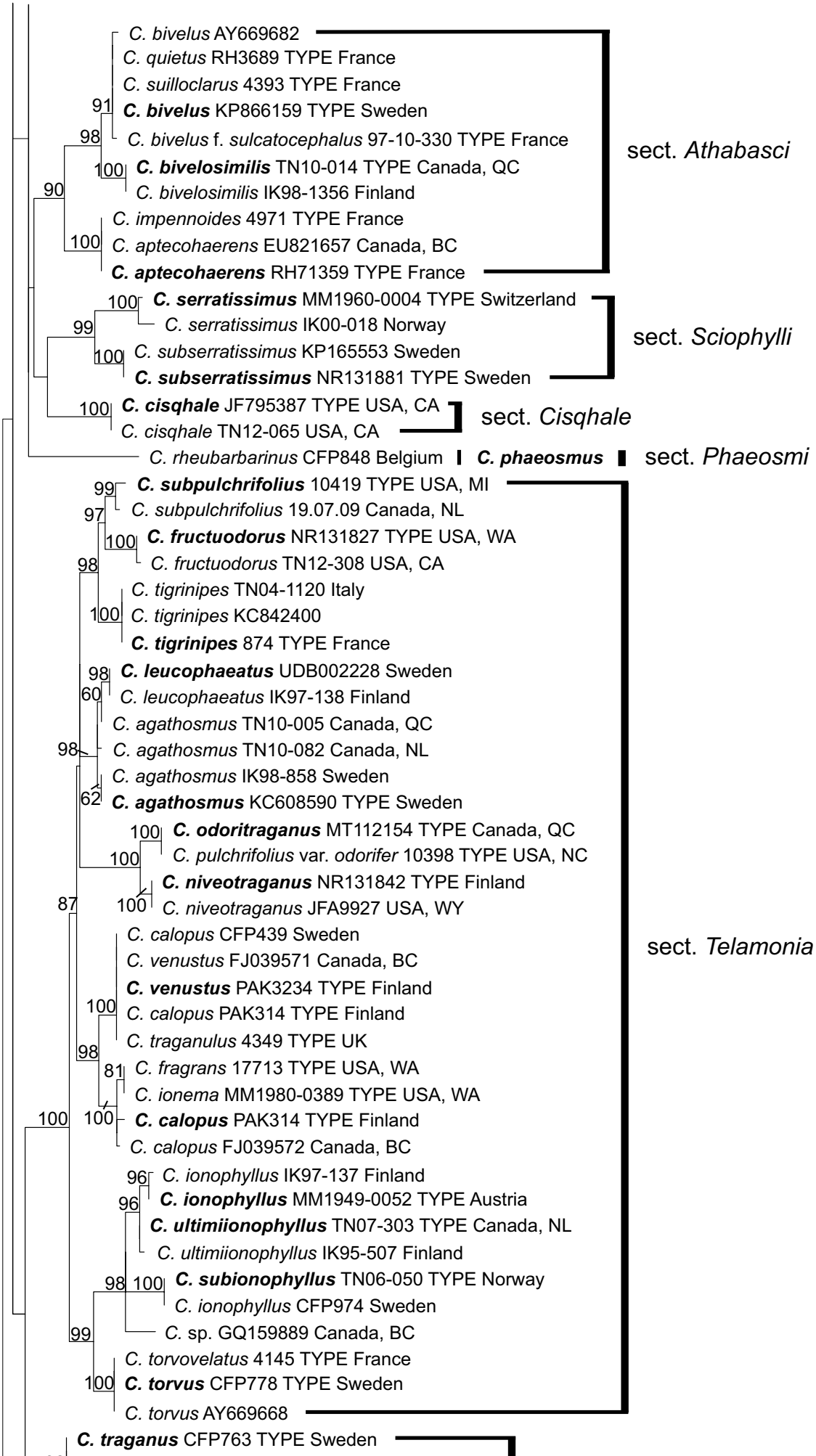

98 C. traganus f. ochraceus JFA9578 TYPE USA, WA

78 C. traganus KC842399 Norway

100 C. venustissimus TN03-938 Sweden

sect. Tragani

C. venustissimus 5371 TYPE Sweden 
Fig. 1 (continued)

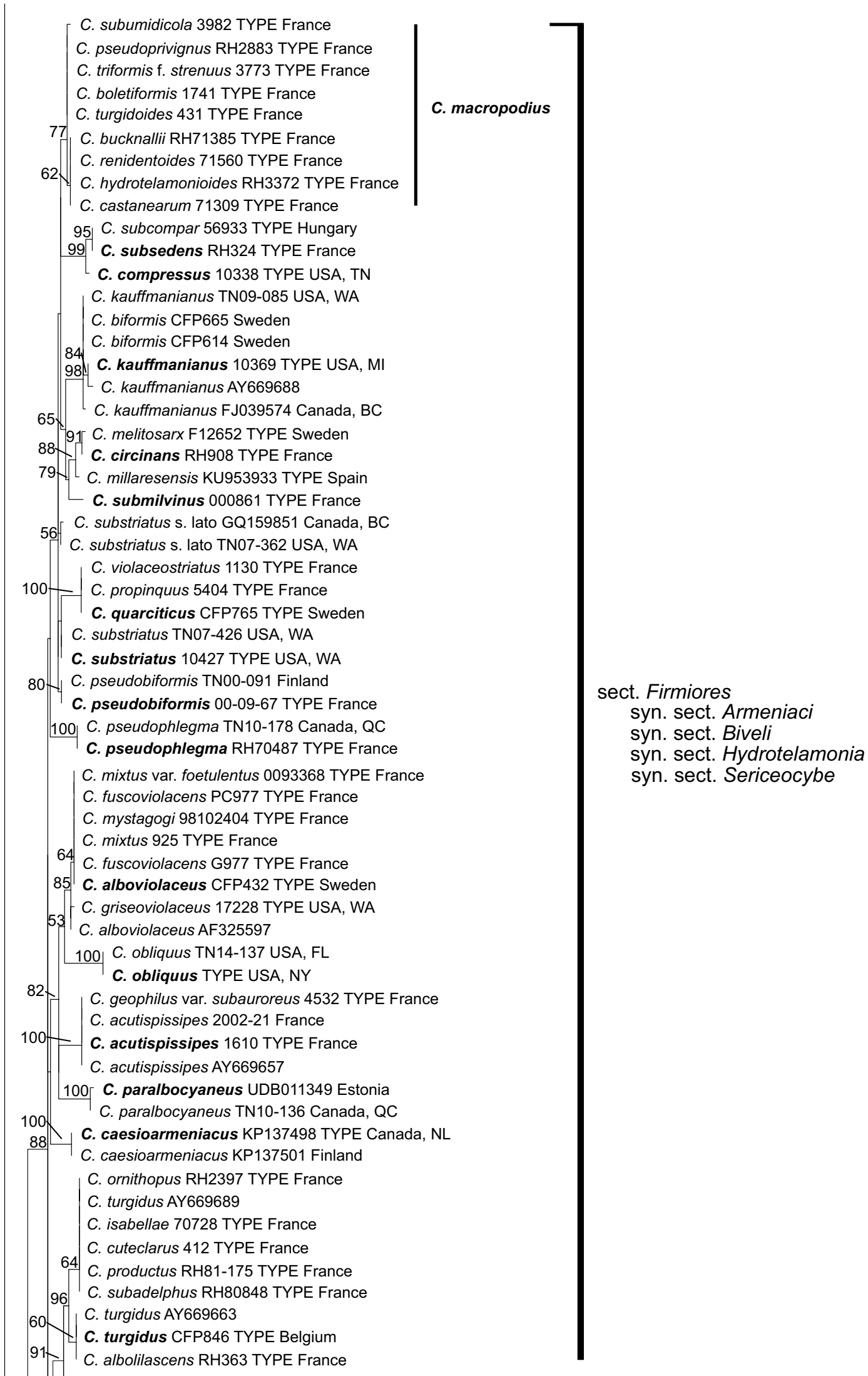


Fig. 1 (continued)

C. armeniacus CFP809 TYPE Sweden

C. hydrobivelus 1213 TYPE France

87 C. privignus 1273 TYPE France

65 C. armeniacus KC842403 Norway

C. carneinatus F22032 TYPE Sweden

c. biveloides $\mathrm{RH} 475$ TYPE France

88 C. gymnopus 2451 TYPE France

994 C. subcarneinatus MF379637 TYPE Norway

C. subcarneinatus 6033545 Finland

C. malachius 1273 TYPE France

C. ochraceus 2138 TYPE USA, NY

100- C. malachius AY669681

C. malachius CFP1198 Sweden

84 C. malachius f. crinitus 4665 TYPE France

C. suberi F16406 TYPE Sweden

C. brunneogriseus F14331 TYPE Sweden

100 C. quarciticus CFP765a Sweden

C. suberi EU821680 Canada, BC

100 C. badiolaevis TN06-301 Finland

C. badiolaevis NR131812 TYPE Sweden

100 C. testaceofolius CFP583 TYPE Sweden

C. biformis TN07-422 USA, WA

64 C. glabrellus s. lato IK04-053 Finland

77 C. glabrellus 10357 TYPE USA, MI

84L C. glabrellus s. lato IK97-756 Sweden

C. glabrellus s. lato AY669692

C. subbalaustinus EU821669 Canada, BC

88 C. subbalaustinus TN09-204 USA, WA

81 C. subbalaustinus CFP926 TYPE Sweden

C. centrirufus JV16764 Finland

100 C. centrirufus AY669693

97 C. centrirufus NR131889 TYPE Finland

98 C. phaeochrous 13309/162 TYPE Switz.

C. phaeochrous TN03-1068 Sweden

100 C. nolaneiformis KJ206491 Sweden

$67 \quad$ C. nolaneiformis NR131833 TYPE Czech

C. vernalisierraensis KX882653 USA, CA

100 C. vernalisierraensis KX882652 TYPE USA, CA

84 - C. ahsii KX882644 TYPE USA, WY

C. ahsii KX882651 USA, WY

109 C. uraceomajalis NR131835 TYPE Hungary

61 C. uraceomajalis 857044 Czech Republic

95 . C. colymbadinus KC842404 Norway

C. colymbadinus KC842404 Norway

${ }_{86}$ C. colymbadinus NR131819 TYPE Sweden

C. colymbadinus KC608592 USA, WA

100 C. subargyronotus KM576360 Austria

95 C. subargyronotus NR131871 TYPE Sweden

C. argyronotus 04-09-84 TYPE France

C. rigidipes KJ206506 Germany

88 C. rigidipes KJ206505 France

100 C. uraceonemoralis KJ206518 Sweden

C. uraceonemoralis NR131836 TYPE Italy

57 99 C. uraceus KC608595 USA, OR

C. uraceus NR131837 TYPE Finland

C. nodosisporus NR131870 TYPE Norway

100 C. rumoribrunsii KX882669 USA, CA

50 C. rumoribrunsii KX882668 USA, CA

99. C. flavobasilis KX882665 USA, MT

C. flavobasilis KX882664 TYPE USA

$86-$ C. vernalishastensis KX882670 TYPE USA, CA

99. C. bridgei KX882661 USA, WA

C. bridgei KX882655 TYPE USA, WA sect. Subbalaustini

1

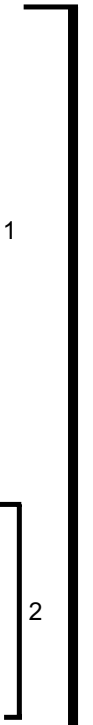

sect. Uracei

syn. sect. Bulliardii

syn. sect. Cinnabarini

syn. sect. Colymbadini

syn. sect. Miniatopodes

1 subsect. Colymbadini

2 subsect. Uracei

3 subsect. Flavobasiles 
Fig. 1 (continued)

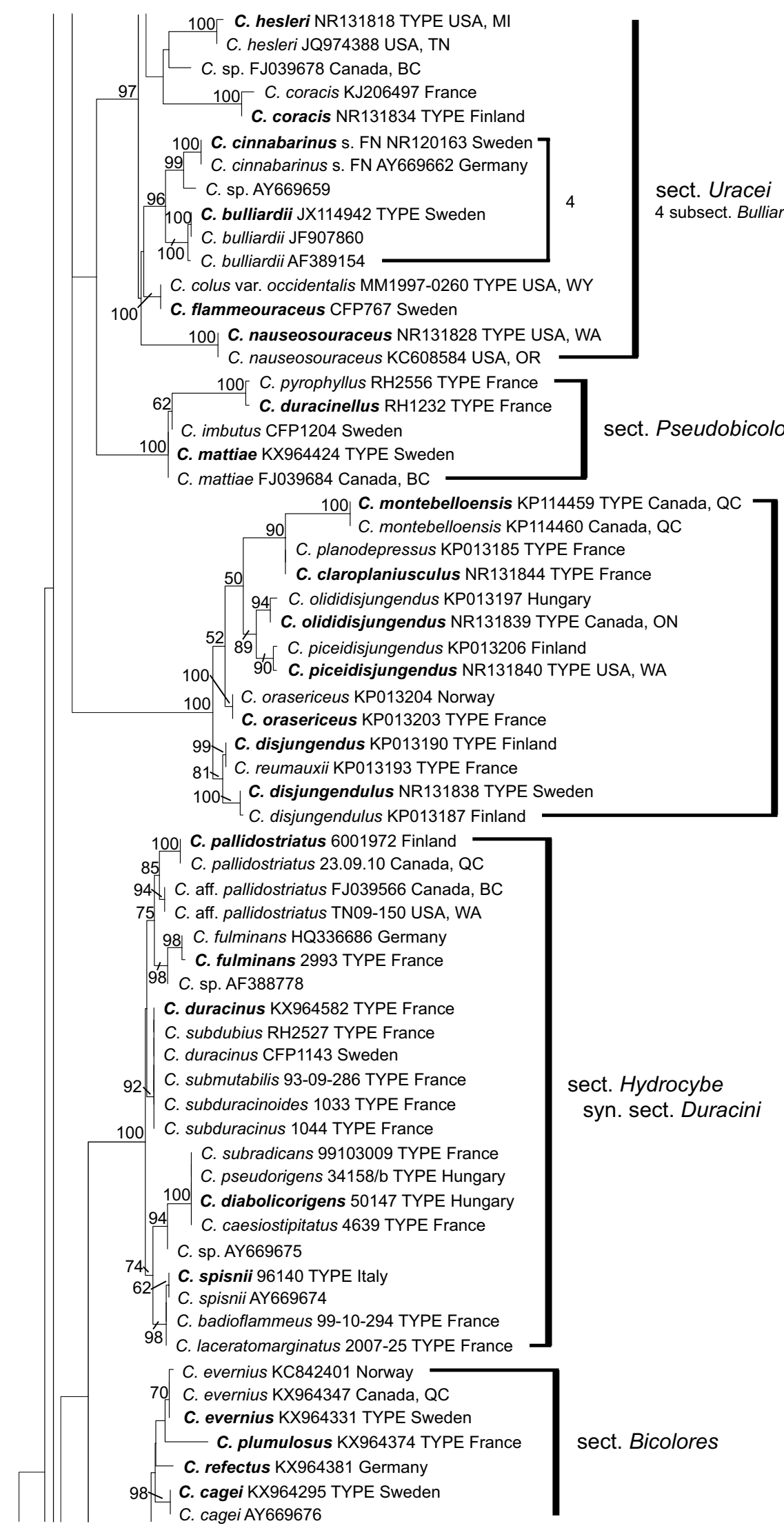

sect. Disjungendi

100 C. pallidostriatus 6001972 Finland

94. C. aff. pallidostriatus FJ039566 Canada, BC

C. aff. pallidostriatus TN09-150 USA, WA

98 C. fulminans HQ336686 Germany

fulminans 2993 TYPE France

C. duracinus KX964582 TYPE France

C. subdubius RH2527 TYPE France

C. submutabilis 93-09-286 TYPE France

C. subduracinoides 1033 TYPE France

C. subradicans 99103009 TYPE France

C. caesiostipitatus 4639 TYPE France

C. sp. AY669675

C. spisnii 96140 TYPE Italy

C. spisnii AY669674

C. badioflammeus 99-10-294 TYPE France

C. laceratomarginatus 2007-25 TYPE France

70 C. evernius KX964347 Canada, QC

C. cagei KX964295 TYPE Sweden

C. cagei AY669676

ect. Hydrocybe

syn. sect. Duracini

sect. Bicolores 
Fig. 1 (continued)

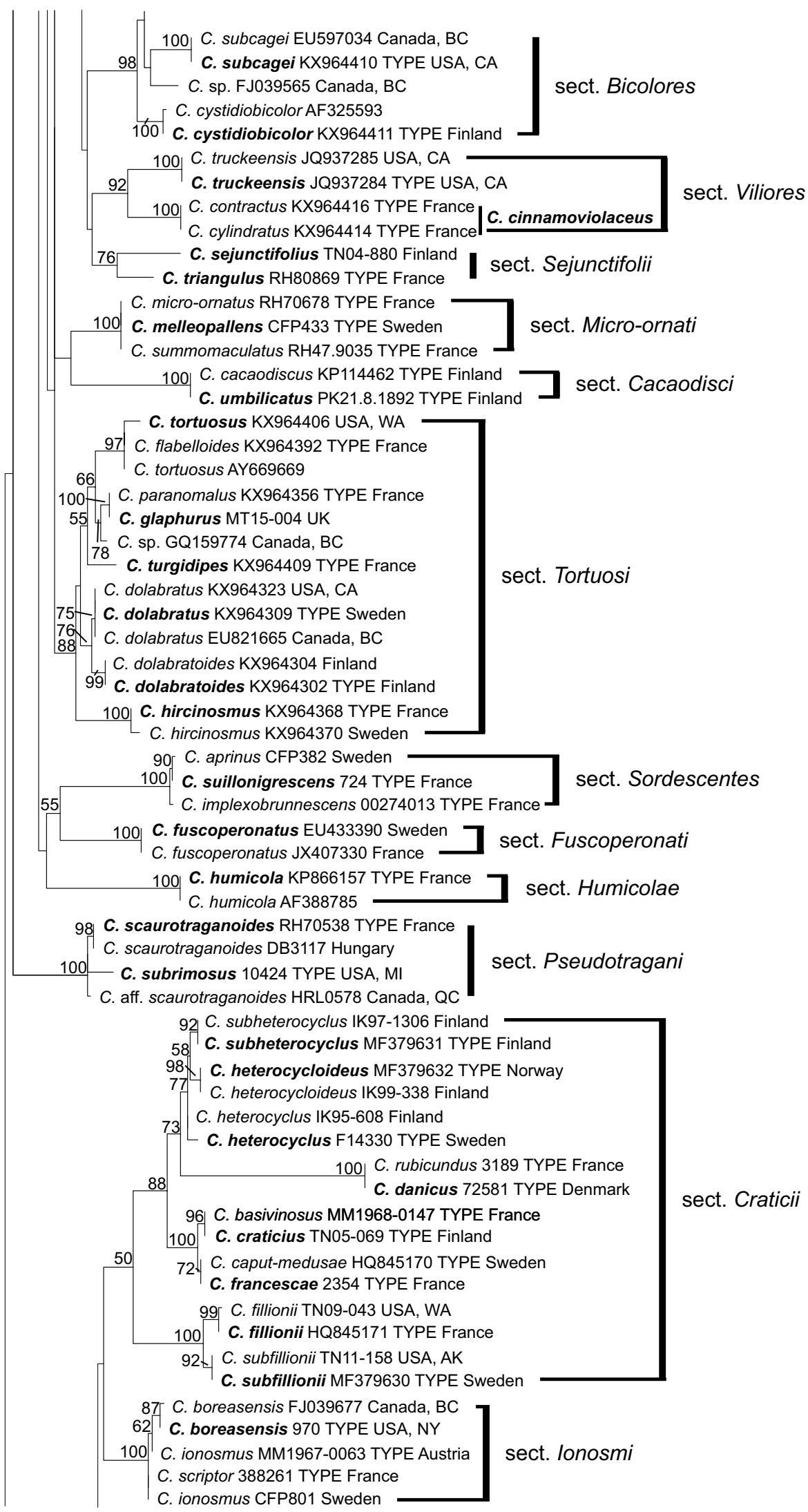


Fig. 1 (continued)

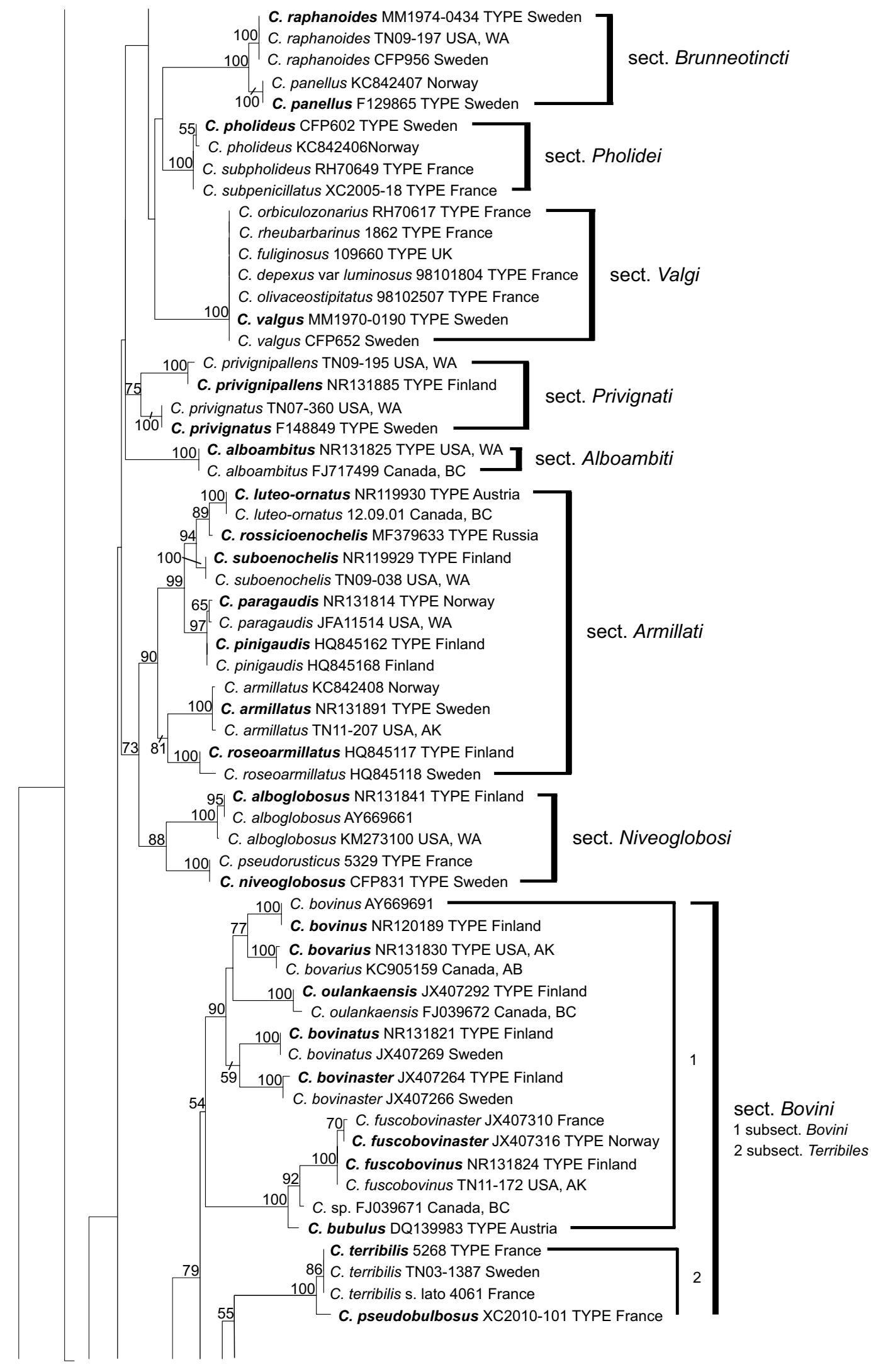


Fig. 1 (continued)

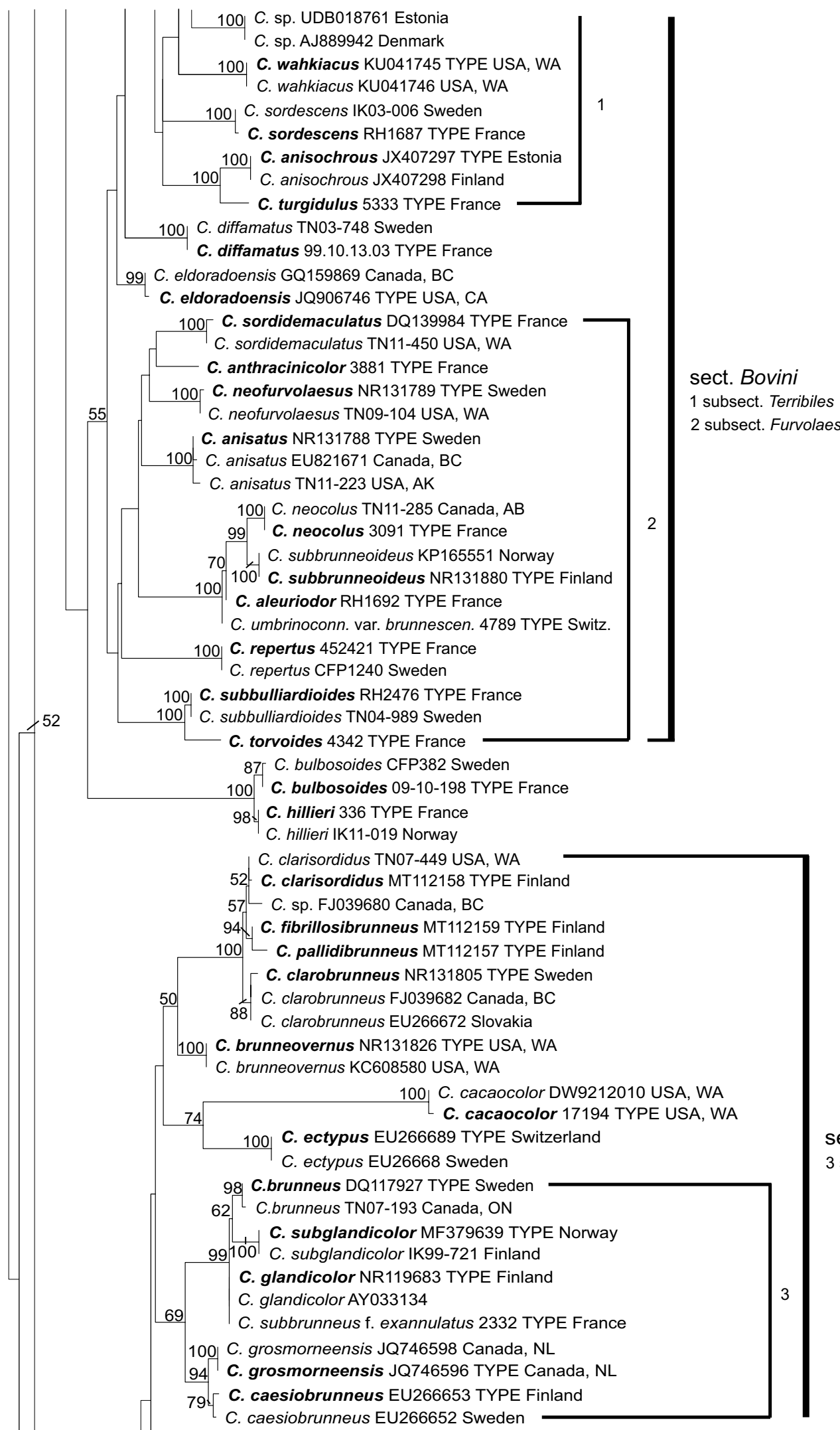

sect. Brunnei 3 subsect. Brunnei 
Fig. 1 (continued)

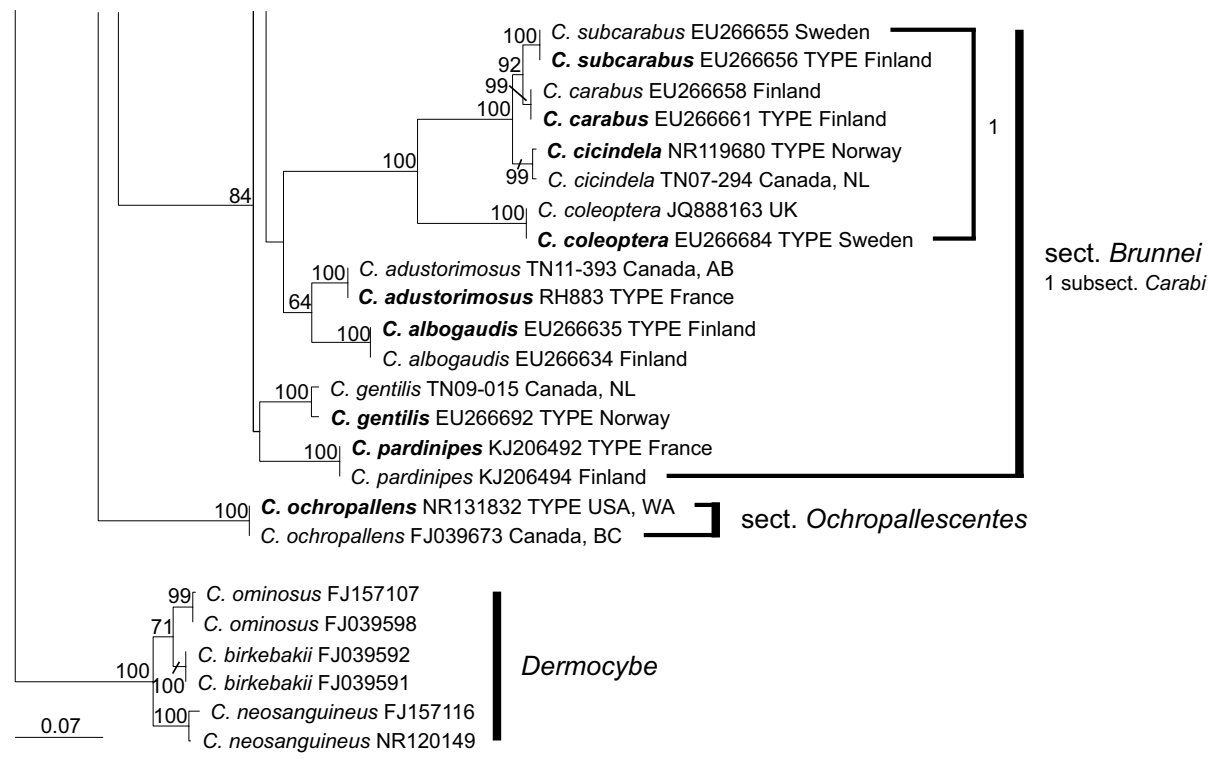

\section{Molecular results}

The phylogenetic tree resulting from the analysis, ITS and LSU regions including binary data from gap coding of the ITS region, is shown in FIG. 1 and a schematic drawing of the relationships of the sections based on the phylogenetic analysis in Fig. 2. Altogether, we recognize $80 \mathrm{sec}-$ tions which all form monophyletic groups in our analysis and examples of the sections are shown in Supplementary Figs. 1-11. We almost entirely used section names with clear identity, i.e., the concept of the type species of a section was well known.

A total of 482 types representing 184 species were successfully sequenced. Of these, about half of the species had one or more synonyms. A significant number of old types were successfully sequenced, 105 types over 50 years old and 18 types over 100 years. All the major Cortinarius taxonomists have described new species that already had an older name, but the portion of younger taxonomic synonyms in terms of the total number of described species (synonym rate) varies among the different authors. Here are synonym rates for authors who have described most of the Telamonia species, based only on morphological characters: Peck $17 \%$, Kauffman 7\%, Smith 40\%, Moser 47\%, Henry 55\%, and French Atlas team 72\%. Current names of Cortinarius species used in this study with their synonyms are listed in Table 1. All the names of the types are listed in alphabetical order in Supplementary Table 1, followed by the current name.

Sometimes it was only possible to amplify part of the ITS region, in most cases it was then the ITS1 region that was succesful. Often in Cortinarius the ITS1 region alone is enough for a proper identification, but especially in the case of small Telamonia species several sister species can have an identical or almost identical ITS1 region. Therefore, all of the unclear cases are marked in Supplementary Tables 1 and 2 with a prefix 'cf.' in the Latin name under the current name.

\section{Taxonomy}

\section{Neo- and epitypifications}

All older names without a type specimen that are included in the Cortinarius subgen. Telamonia key in Funga Nordica (Niskanen et al. 2012) and not yet typified are typified here with the exceptions of $C$. paleaceus $\mathrm{Fr}$. and $C$. miniatopus J.E. Lange (not included in Niskanen et al. 2012) and $C$. psammocephalus (Bull.) Fr. (nomen dubium, no type proposed). In addition, for $C$. colus Fr. an epitype that differs from the current use of the name is proposed, and in the case of C. alboviolaceus (Pers.) Fr., C. flabellus (Fr.) Fr. and $C$. hinnuleus Fr. the best fitting candidate from two or more avalaible ones in a species group was selected. For C. anthracinus Fr. and C. cinnabarinus Fr. neotypes have been chosen by Høiland (1983) but were not sequenced in this study. Altogether, neotypes for 11 species originally described by Fries, Liljeblad and Persoon are proposed as well as epitypes for 9 species described by Fries, Lange, Persoon, Quélet and Schaeffer. Citations of descriptions and illustrations of the species are provided. Notes under the name have only been added if our typification does not fit with the protologue and/or differs from the current use of the name in Niskanen et al. (2012). Synonyms are based on 


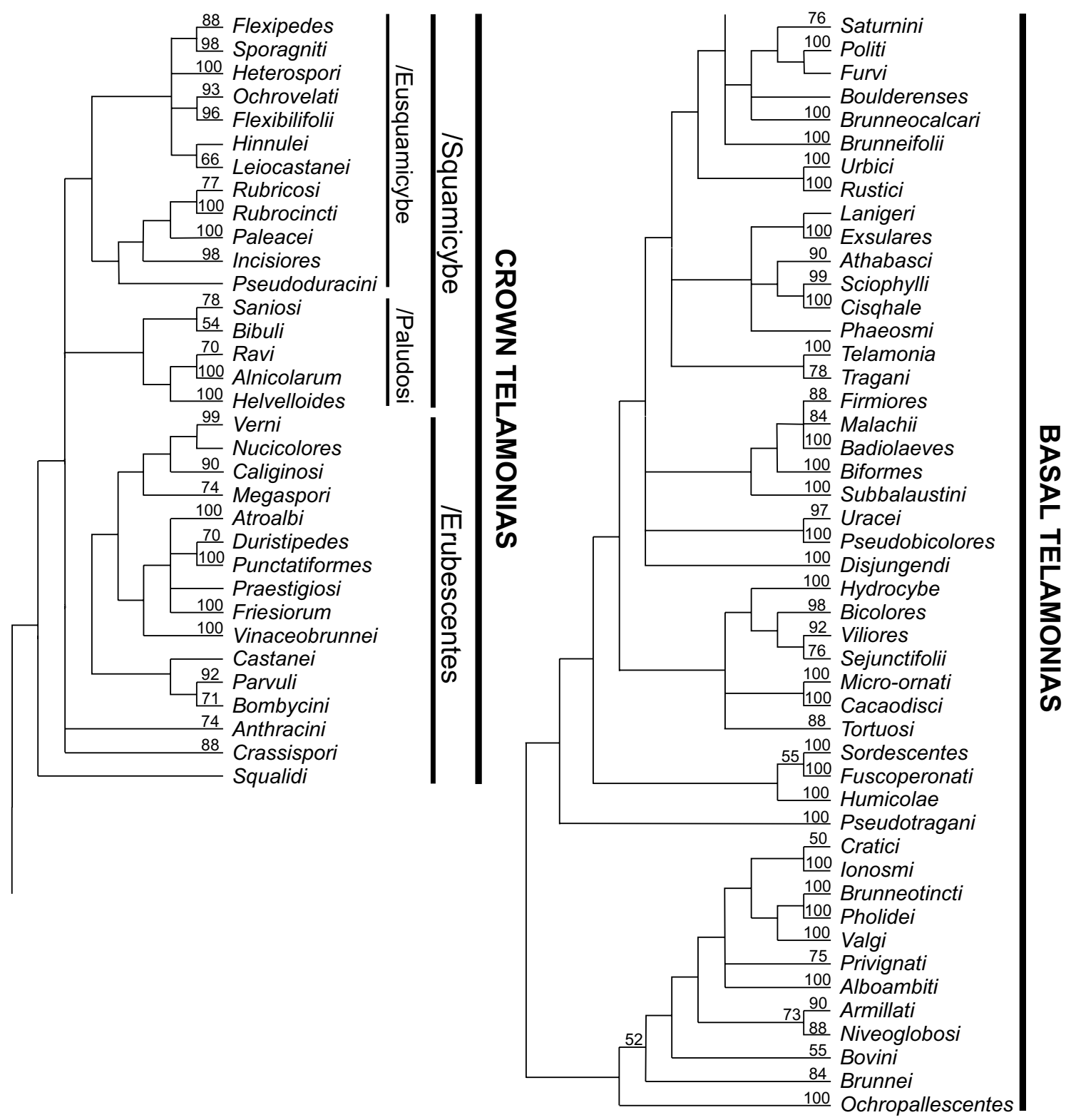

Fig. 2 A schematic drawing of the relationships of the 80 sections of Cortinarius subgen. Telamonia accepted in this study. The drawing is based on our phylogenetic analysis with bootstrap values greater than 50\% gained in the analysis indicated above branches

DNA studies of the type specimens and the information on the types is presented in Table 1.

Cortinarius alboviolaceus (Pers.) Fr., Epicr. syst. mycol. (Upsaliae): 280 (1838) [1836-1838]

Basionym: Agaricus alboviolaceus Pers., Syn. meth. fung. (Göttingen) 2: 286 (1801): sanctioned in Fr., Syst. mycol. 1: 218 (1821).

Type: Sweden, Härjedalen, Storsjö sn, Flatruet, in subalpine zone with Betula pubescens, 16 Aug 1986, coll. H.
Lindström et al. CFP 432, F41130 (S, neotypus hic designatus, IF 557454), GenBank No. MT934857 (ITS).

Illustration. Brandrud et al. (1989: pl. A59).

Descriptions of the species. Brandrud et al. (1989: pl. A59), Niskanen et al. (2012).

Notes-Currently, we know three species in Europe that fit into Fries' description of C. alboviolaceus: C. acutispissipes Rob. Henry, C. alboviolaceus s. Brandrud et al. (1989) $\&$ Niskanen et al. (2012), and C. paralbocyaneus Eyssart. Based on the current data C. alboviolaceus s. auctores has the widest distribution of the three species and is also the 
Table 1 Current names of the Cortinarius species accepted in this study with their synonyms

\begin{tabular}{ll}
\hline Current name & Younger synonyms
\end{tabular}

C. aavae Liimat. \& Niskanen 2012

C. acutispissipes Rob. Henry 1981

C. adustorimosus Rob. Henry 1988

C. ahsii McKnight 1975

C. alboadustus Bidaud 2012

C. alboambitus Niskanen, Liimat. \& Ammirati 2013

C. albogaudis Kytöv., Niskanen \& Liimat. 2009

C. alboglobosus Kytöv., Liimat., Niskanen \& Ammirati 2014

C. albolens Bidaud, Carteret \& Reumaux 2012

C. albosericeus Ammirati, Beug, Liimat., Niskanen \& O. Ceska 2016

C. alboviolaceus (Pers.) Fr. 1838

C. aleuriodor Rob. Henry 1981

C. americanus A.H. Sm. 1934

C. ammophiloides Bohus 1979

C. andreae H. Lindstr. 1999 s. lato

C. angelesianus A.H. Sm. 1944

C. angustisporus Kytöv., Niskanen \& Liimat. 2014

C. anisatus H. Lindstr., Kytöv. \& Niskanen 2005

C. anisochrous Kytöv., Liimat., Niskanen \& H. Lindstr. 2013

C. anthracinicolor Reumaux 2001

C. anthracinus (Fr.) Sacc. 1887 s. Funga Nordica

C. aptecohaerens Rob. Henry 1983

C. argyronotus Bidaud 2008

C. armeniacus (Schaeff.) Fr. 1838

C. armillatus (Fr.) Fr. 1838

C. atroalbus M.M. Moser 1993

C. atrocaeruleus M.M. Moser 1967

C. auroripes Carteret \& Reumaux 2001

C. badioflavidus Ammirati, Beug, Niskanen, Liimat. \& Bojantchev 2016

C. badiolaevis Niskanen, Liimat., Mahiques, Ballarà \& Kytöv. 2011

C. badiovestitus M.M. Moser 1968

C. bibulus Quél. 1881

C. biformis Fr. 1838

C. bistreoides Kauffman 1921

C. biveloides Rob. Henry 1948

C. bivelosimilis Kytöv., Niskanen \& Liimat. 2017

C. bivelus (Fr.) Fr. 1838
C. geophilus var. subauroreus Bidaud, Moënne-Locc. \& Reumaux 2002

C. fuscoviolascens Reumaux 1990; C. mixtus Reumaux 2002; C. mixtus var. foetulentus Carteret, Moënne-Locc. \& Reumaux 2002; C. mystagogi Carteret \& Reumaux 2002; C. radicatoviolaceus Rob. Henry 1981; C. griseoviolaceus A.H. Sm. 1944

C. perrinii Rob. Henry \& Ramm 1989; C. umbrinoconnatus var. brunnescentipes Bidaud, Moënne-Locc., Reumaux \& Rob. Henry 2000

C. sphagnicola Carteret \& Reumaux 2004

C. ochrorufus Moënne-Locc. \& Fillion 2001; C. punctatorum MoënneLocc. \& Fillion 2012; C. salicetophilus Bidaud \& Fillion 2012; C. subfistularis Fillion 2012; C. tener Carteret 2001; C. nigromaculatus Carteret 2012

C. sporagnitus Bidaud 2010

C. angelesianus var. elakatopus M.M. Moser 2001; C. strobilaceus M.M. Moser 1967; C. tubulipes J. Favre 1960

C. danili Rob. Henry 1943; C. ignifluus Bidaud 1994; C. josserandii Bidaud 1994; C. procalans Moënne-Locc. \& Reumaux 1994; C. subanthracinus Rob. Henry 1944;

C. impennoides Bidaud, Moënne-Locc. \& Reumaux 2000

C. hydrobivelus Rob. Henry \& Reumaux 2010; C. privignorum Rob. Henry 1985; C. privignus (Fr.) Fr. 1838

C. atroalbus var. nigripes M.M. Moser 1993; C. ignotissimus Bidaud 2012; C. paludophilus Carteret \& Reumaux 2001

C. carminipes Moënne-Locc. \& Reumaux 1988; C. erythrinellus Reumaux 1988; C. insignitus Reumaux 2004

C. subgracilior Bidaud \& Carteret 2008

C. testaceofolius H. Lindstr. \& Soop 1995

C. carneinatus Soop 2002; C. gymnopus Rob. Henry 1983

C. amphibalaustius Rob. Henry 1983; C. bivelus f. sulcatocephalus Bidaud 2010; C. insolitus Rob. Henry 1981; C. quietus Rob. Henry 1977; C. suilloclarus Reumaux 2002 
Table 1 (continued)

\begin{tabular}{|c|c|}
\hline Current name & Younger synonyms \\
\hline \multicolumn{2}{|l|}{ C. bombycinus Mahiques \& Burguete 2001} \\
\hline \multicolumn{2}{|l|}{ C. bonamei Rob. Henry 1970} \\
\hline C. boreasensis A.H. Sm. 1944 & $\begin{array}{l}\text { C. ionosmus M.M. Moser, Nespiak \& Schwöbel 1969; C. scriptor } \\
\text { Kühner } 1989\end{array}$ \\
\hline \multicolumn{2}{|l|}{ C. boreotrichus Kytöv., Niskanen \& Liimat. 2017} \\
\hline \multicolumn{2}{|l|}{ C. boulderensis A.H. Sm. 1944} \\
\hline \multicolumn{2}{|l|}{ C. bovarius Liimat. \& Niskanen 2013} \\
\hline \multicolumn{2}{|l|}{ C. bovinaster Niskanen, Kytöv. \& Liimat. 2013} \\
\hline \multicolumn{2}{|l|}{ C. bovinatus Kytöv., Liimat., Niskanen \& H. Lindstr. 2013} \\
\hline \multicolumn{2}{|l|}{ C. bovinus Fr. 1838} \\
\hline \multicolumn{2}{|c|}{ C. bridgei Ammirati, Niskanen, Liimat., Bojantchev, \& Fang 2017} \\
\hline \multicolumn{2}{|l|}{ C. brunneifolius Kytöv., Niskanen \& Liimat. 2008} \\
\hline \multicolumn{2}{|l|}{ C. brunneocalcarius Niskanen, Liimat. \& Kytöv. 2012} \\
\hline \multicolumn{2}{|l|}{ C. brunneoclarus Niskanen, Kytöv. \& Liimat. 2020} \\
\hline \multicolumn{2}{|l|}{ C. brunneovernus Niskanen, Liimat. \& Ammirati 2013} \\
\hline C. brunneus (Pers.) Fr. 1838 & C. brunneus var. incommixtus Bidaud 2009 \\
\hline \multicolumn{2}{|l|}{ C. bubulus Liimat., Kytöv. \& Niskanen 2020} \\
\hline \multicolumn{2}{|l|}{ C. bulbosovolvatus Rob. Henry \& Contu 1985} \\
\hline C. bulliardii (Pers.) Fr. 1838 & C. colus Fr. 1838; C. georgianae Moënne-Locc. 1994 \\
\hline \multicolumn{2}{|l|}{ C. cacaocolor A.H. Sm. 1944} \\
\hline \multicolumn{2}{|l|}{ C. caesioarmeniacus Kytöv., Niskanen \& Liimat. 2014} \\
\hline \multicolumn{2}{|l|}{ C. caesiobrunneus Kytöv., Niskanen \& Liimat. 2009} \\
\hline \multicolumn{2}{|l|}{ C. cagei Melot 1990} \\
\hline \multicolumn{2}{|l|}{ C. californicus A.H. Sm. 1939} \\
\hline \multicolumn{2}{|l|}{ C. caliginosus Bidaud, Moënne-Locc. \& Reumaux 2000} \\
\hline C. calopus P. Karst. 1881 & C. fragrans A.H. Sm. 1944; C. ionema M.M. Moser \& Ammirati 1996 \\
\hline C. campester Reumaux 2001 & C. malefidus Moënne-Locc. 2001; C. porphyreticus Moënne-Locc. 2001 \\
\hline \multicolumn{2}{|l|}{ C. canaliculatus Bidaud \& Carteret 2008} \\
\hline \multicolumn{2}{|l|}{ C. carabus Kytöv., Niskanen \& Liimat. 2009} \\
\hline \multicolumn{2}{|l|}{ C. carbunculus H. Lindstr. \& H. Markl. 2010} \\
\hline C. castaneopallidus Carteret 2004 & $\begin{array}{l}\text { C. franceschinii Consiglio, D. Antonini \& M. Antonini 2006; C. } \\
\text { olivaceomarginatus Carteret 2012; C. pseudobavaricus Bidaud \& } \\
\text { Reumaux } 2012\end{array}$ \\
\hline C. castaneus (Bull.) Fr. 1838 & $\begin{array}{l}\text { C. fallaciosus Bidaud 2001; C. kunicensis var. caespitosus Moënne- } \\
\text { Locc. 2001; C. robertii Moënne-Locc. \& Reumaux 1988; C. subodo- } \\
\text { ratus Bidaud } 2001\end{array}$ \\
\hline \multicolumn{2}{|l|}{ C. centrirufus Kytöv., Niskanen \& Liimat. 2014} \\
\hline C. chevassutii Rob. Henry 1982 & C. subsordescens Rob. Henry 1985 \\
\hline C. cicindela Kytöv., Niskanen \& Liimat. 2009 & \\
\hline C. cinereobrunneolus Chevassut \& Rob. Henry 1982 & \\
\hline C. cinnamoviolaceus M.M. Moser 1968 & \\
\hline C. circinans Rob. Henry 1985 & C. melitosarx Soop 1999 \\
\hline C. cisqhale Bojantchev 2013 & \\
\hline C. cistopulchripes Bidaud 2004 & \\
\hline $\begin{array}{l}\text { C. clarobrunneus (H. Lindstr. \& Melot) Niskanen, Ky } \\
2009\end{array}$ & \\
\hline C. claroplaniusculus Rob. Henry 1983 & \\
\hline C. clarosordidus Niskanen, Kytöv. \& Liimat. 2020 & \\
\hline C. coccineus Reumaux 1994 & \\
\hline C. coleoptera H. Lindstr. \& Soop 1999 & \\
\hline C. colorius (Bidaud) Niskanen, Dima \& Liimat. 2020 & \\
\hline
\end{tabular}


Table 1 (continued)

Current name
C. colymbadinus Fr. 1838
C. compressus A.H. Sm. 1944
C. comptulus M.M. Moser 1968

C. confirmatus Rob. Henry 1983

C. coracis Kytöv., Niskanen, Liimat. \& Dima 2014

C. corvinus Reumaux 2012

C. crassisporus Kytöv., Niskanen \& Liimat. 2014

C. craticius Fr. 1838

C. cucumisporus M.M. Moser 1967

C. cystidiobicolor Liimat. \& Niskanen 2017

C. danicus Høil. 1983

C. deceptivissimus Reumaux 1984

C. decipiens (Pers.) Fr. 1838

C. decipientoides Moënne-Locc. \& Reumaux 1988

C. denigratus Ammirati, Beug, Niskanen, Liimat. \& O. Ceska 2016

C. desertorum (Velen.) G. Garnier 1991

C. diabolicorigens Bohus 1976

C. diffamatus Carteret 2012

C. diffractosuavis Chevassut \& Rob. Henry 1978

C. diosmoides Rob. Henry 1989

C. diosmus Kühner 1955

C. disjungendulus Kytöv., Liimat. \& Niskanen 2014

C. disjungendus P. Karst. 1893

C. distortus Kauffman 1932

C. dolabratoides Kytöv., Carteret, Bidaud, Liimat., Niskanen, Bellanger, Dima, Reumaux \& Ammirati 2017

C. dolabratus Fr. 1838

C. duboisensis Ammirati, Beug, Niskanen \& Liimat. 2016

C. dumetorum J. Favre 1960

C. duracinellus Rob. Henry 1970

C. duracinus Fr. 1838

C. duristipes Kytöv., Niskanen \& Liimat. 2014

C. ectypus J. Favre 1960

C. elaphinicolor Carteret 2004

C. eldoradoensis Bojantchev 2013
Younger synonyms

C. fistularioides Reumaux, Bidaud \& Fillion 2001; C. griseosulcatus Carteret 2004; C. hemitrichus var. americanus A.H. Sm. 1944; C. inolens var. parvinolens Bidaud \& Carteret 2010; C. jacobii Bidaud, Moënne-Locc. \& Reumaux 2001; C. laniatus Rob. Henry 1983; C. sublatisporus Svrček 1968

C. basivinosus M.M. Moser 1996

C. rubicundus Bidaud, Moënne-Locc. \& Reumaux 1994

C. damascenoides Bidaud 2008

C. anthracinoides Rob. Henry 2001; C. argumentosus Moënne-Locc. \& Reumaux 2000; C. decipiens f. saliceticola Reumaux \& Carteret 2001; C. maculatophyllus Bidaud 2012; C. washingtonensis A.H. Sm. 1939

C. alutaceogrisescens Bidaud 1997; C. derelictus Reumaux 2001; C. rubellopes var. pudoricolor Bidaud, Moënne-Locc. \& Reumaux 2001

C. ammophilus A. Pearson 1946; C. diasemospermus var. leptospermus H. Lindstr. 1998; C. difficillimus Carteret 2012; C. goniosporus Carteret 2004; C. pertristis J. Favre 1955; C. subdepressus Carteret 2012; C. subrigidus Bidaud, Carteret \& Reumaux 2010; C. friesianus Carteret \& Reumaux 2001

C. caesiostipitatus Reumaux 2008; C. pseudorigens Bohus 1976; C. subradicans Carteret \& Reumaux 2008

C. diosmoides Rob. Henry 1989

C. reumauxii Rob. Henry 1996

C. parasuillus Reumaux 2010; C. pseudocalopus Reumaux 2010; C. subcurtipes Bidaud 2010; C. submelleopallens Rob. Henry 1981

C. pyrophyllus Rob. Henry 1970

C. subdubius Rob. Henry 1970; C. subduracinoides Moënne-Locc. \& Reumaux 2008; C. subduracinus Bidaud \& Moënne-Locc. 2008; $C$. submutabilis Bidaud \& Carteret 2008 
Table 1 (continued)

Current name
C. epipurrus Chevassut \& Rob. Henry 1978
C. evernius (Fr.) Fr. 1838
C. exitiosus Bidaud, Moënne-Locc. \& Reumaux 2001
C. expallens M.M. Moser 1993
C. exsularis Garrido-Ben., Ballarà \& Mahiques 2016
C. fagetorum M.M. Moser 1967
C. falsosus Moënne-Locc. \& Reumaux 2001
C. famatus Moënne-Locc. \& Reumaux 2001
C. ferrugineovelatus Kytöv. Liimat. \& Niskanen 2014
C. fibrillosobrunneus Kytöv., Niskanen \& Liimat. 2020
C. fillionii Bidaud, Moënne-Locc. \& Reumaux 1995
C. flabellus (Fr.) Fr. 1838

Current name Younger synonyms

C. hinnuleoradicatus Bidaud, Moënne-Locc. \& Reumaux 1997; C. pallescens Moënne-Locc. \& Reumaux 1997; C. pseudosafranopes Moënne-Locc. \& Reumaux 1997; C. subgrisescens Bidaud, MoënneLocc. \& Reumaux 1997

C. mucronatus M.M. Moser \& McKnight 1987, nom. illegit.; C. hemitrichus f. improcerus J. Favre 1955; C. gossypinus H. Lindstr. 2010

C. fagetorum M.M. Moser 1967

C. griseophyllus Reumaux 2001; C. griseovioleipes Moënne-Locc. \& Reumaux 2001; C. recedens Bidaud, Moënne-Locc. \& Reumaux 2001

C. biformis var. dilatus Bidaud, Carteret \& Reumaux 2012

C. flabelliformis Bidaud 2010; C. flabellus f. iners Bidaud 2010; C. furfuraceus Bidaud 1997; C. fuscoruber Reumaux 2001; C. gurdus Carteret 2012; C. pseudodepressus Carteret \& Reumaux 2010

C. flammeouraceus Niskanen, Kytöv., Liimat., Dima \& Ammirati 2020 C. colus var. occidentalis M.M. Moser 2002

C. flavobasilis Peintner, Kuhnert-Finkernagel, Cripps \& Ammirati 2017

C. flexibilifolius Carteret 2004

C. flexipes (Pers.) Fr. 1838

C. fragrantissimus Ammirati, Beug, Liimat., Niskanen \& O. Ceska 2016

C. francescae Reumaux 1992

C. fructuodorus Niskanen, Liimat. \& Ammirati 2013

C. fulminans Moënne-Locc. \& Reumaux 2008

C. fulvoisabellinus Rob. Henry 1941

C. fulvopaludosus Kytöv., Niskanen \& Liimat. 2017

C. furfurellus Peck 1880

C. furvoumbrinus Liimat., Niskanen \& Kytöv. 2014

C. furvus Liimat., Niskanen \& Kytöv. 2014

C. fuscescens Kytöv., Niskanen \& Liimat. 2014

C. fuscoalbus Kytöv., Niskanen \& Liimat. 2017

C. fuscobovinaster Kytöv., Liimat., Niskanen \& H. Lindstr. 2013

C. fuscobovinus Kytöv., Niskanen \& Liimat. 2013

C. fuscodiscus A.H. Sm. 1944

C. fuscoflexipes M.M. Moser \& McKnight 1987

C. fuscogracilescens A. Favre 2009

C. fuscoperonatus Kühner 1953

C. fuscoumbrinus Liimat., Niskanen \& Kytöv. 2014

C. fuscovelatus Kytöv., Niskanen \& Liimat. 2014

C. gallurae D. Antonini, M. Antonini \& Consiglio 2005

C. gentilis (Fr.) Fr. 1838

C. gentilissimus A.H. Sm. 1939

C. geraniolens Bidaud 2010 s. lato

C. glabrellus Kauffman 1907

C. glandicolor (Fr.) Fr. 1838

C. glaphurus Chevassut \& Rob. Henry 1982
C. olivaceobrunneus Reumaux 2012; C. privus Reumaux 2004; C. subfuscodiscus Reumaux 2004

C. paleifer Svrček 1968

C. caput-medusae H. Lindstr. 1998
C. subcarcharias Bidaud 2010

C. leiopus Rob. Henry 1981

C. subbrunneus f. exannulatus Moënne-Locc. 2009

C. paranomalus Rob. Henry 1992 
Table 1 (continued)

Current name
C. griseocarneus Carteret 2010
C. grosmorneënsis Liimat. \& Niskanen 2012
C. gualalaensis Bojantchev 2013
C. helodes M.M. Moser, Matheny \& Daniele 2001
C. helvelloides (Bull.) Fr. 1838
C. hemitrichoides Bidaud \& Moënne-Locc. 2010
C. hemitrichus (Pers.) Fr. 1838
C. heparinus Kytöv., Niskanen \& Liimat. 2020
C. hesleri Ammirati, Niskanen, Liimat. \& Matheny 2013
C. heterocycloideus Kytöv., Niskanen \& Liimat. 2017
C. heterocyclus Soop 1990
C. heterodepressus Kytöv., Niskanen \& Liimat. 2017
C. heterosporus Bres. 1889
C. hillieri Rob. Henry 1938
C. hinnuleoarmillatus Reumaux 1989
C. hinnuleocervinus Niskanen, Liimat. \& Ammirati 2017
C. hinnuleus Fr. 1838
C. hircinosmus Moënne-Locc. 2002
C. hirtus (Velen.) G. Garnier 1991

C. humicola (Quél.) Maire 1911

C. hydrotelamonioides Rob. Henry 1970

C. imbutus Fr. 1838

C. impolitus Kauffman 1918

C. incisior Bidaud, Moënne-Locc. \& Reumaux 1997

C. inconspicuus J. Favre 1955

C. iners (Bidaud) Liimat., Dima \& Niskanen 2020

C. intempestivus Moënne-Locc. \& Reumaux 2001

C. ionophyllus M.M. Moser 1968

C. jacobi-langei Bidaud 2008

C. kauffmanianus A.H. Sm. 1933

C. lacustris Moënne-Locc. \& Reumaux 1997

C. laniger Fr. 1838

C. leiocastaneus Niskanen, Liimat. \& Soop 2008

C. leucophaeatus Rob. Henry 1985

C. lindstroemii Niskanen, Kytöv. \& Liimat. 2020

C. lucorum (Fr.) Berger 1846
Younger synonyms

C. atripes Reumaux 2012

C. fusisemen Reumaux 2000; C. milvinoides Carteret \& Reumaux 2010;

C. paleaceus Fr. 1838; C. roseohemitrichus Carteret \& Reumaux 2010

C. bohemicus f. subheterosporus Bidaud 2010

C. aureifer Reumaux 1989

C. distans var. olympianus A.H. Sm. 1944

C. tigris Bidaud 1997

C. querculus Moënne-Locc. \& Reumaux 2001; C. punctatoides Reumaux 2012; Telamonia sanguinescens Velen. 1939; C. similigenus Moënne-Locc. \& Reumaux 2001; C. subargyropus Bidaud, MoënneLocc. \& Reumaux 2001; C. substemmatus Moënne-Locc. \& Reumaux 2001

C. boletiformis Bidaud \& Reumaux 2010; C. bucknallii Reumaux 2010; C. castanearum Rob. Henry 1981; C. macropodius Rob. Henry 1961 Nom. inval.; C. pseudoprivignus Rob. Henry 1985; C. renidentoides Rob. Henry 1981; C. subumidicola Bidaud, Moënne-Locc. \& Reumaux 2000; C. triformis f. strenuus Rob. Henry 1981; C. turgidoides Rob. Henry 1981

C. subacutus A.H. Sm. 1944

C. perzonatus Reumaux 2004; C. spurcatus Moënne-Locc. \& Reumaux 2001

C. erubescens M.M. Moser 1968

C. cristatosporus Reumaux 2004

C. altae-herbae Moënne-Locc. \& Reumaux 1999

C. laniger f. macrosemen Bidaud, Carteret \& Reumaux 2010

C. flabellus f. biolens Bidaud 2010 
Table 1 (continued)

\section{Current name}

C. luridis Rob. Henry 1969

C. luteo-ornatus (M.M. Moser) Bidaud, Moënne-Locc. \& Reumaux 1995

C. malachius (Fr.) Fr. 1838

C. mallaensis Kytöv., Niskanen \& Liimat. 2020

C. mattiae Soop 2010

C. megacystidiosus Reumaux 2012

C. melleopallens (Fr.) Britzelm. 1892

C. milvinicolor Moënne-Locc. \& Reumaux 1997

C. miniatopus J.E. Lange 1940

C. minusculus Liimat. \& Niskanen 2019

C. miwok Bojantchev 2013

C. montebelloensis Niskanen \& Liimat. 2014

C. mucicola A.H. Sm. 1944

C. murinascens Kytöv., Niskanen \& Liimat. 2014

C. nauseosouraceus Niskanen, Liimat. \& Ammirati 2013

C. neocolus Reumaux \& Sasia 2011

C. neofallax Carteret \& Reumaux 2004

C. neofurvolaesus Kytöv., Niskanen, Liimat. \& H. Lindstr. 2005

C. nigrellus Peck 1873

C. nigrocuspidatus Kauffman 1921

C. niveoglobosus H. Lindstr. 1992

C. niveotraganus Kytöv., Niskanen \& Liimat. 2014

C. nodosisporus Kytöv., Niskanen \& Liimat. 2014

C. nolaneiformis (Velen.) Dima, Niskanen \& Liimat. 2014

C. nucicolor Liimat., Niskanen \& Kytöv. 2014

C. obliquus Peck 1902

C. ochropallens Liimat., Niskanen \& Ammirati 2013

C. ohlone Bojantchev 2013

C. olididisjungendus Liimat., Niskanen, Dima \& Kytöv. 2014

C. olivaceofulvus Kauffman \& A.H. Sm. 1933

C. orasericeus Rob. Henry 1983

C. oulankaënsis Kytöv., Niskanen, Liimat. \& H. Lindstr. 2013

C. pallidostriatus Rob. Henry 1968

C. paludosaniosus Liimat., Niskanen, Dima \& Ammirati 2017

C. panellus Soop 2009

C. pangloius M.M. Moser 1969

C. paragaudis Fr. 1838

C. paralbocyaneus Eyssart. 2002

C. pardinipes Romagn. 1977
Younger synonyms

C. aciculisporus Moënne-Locc. 1997; C. armillifer Moënne-Locc. \& Reumaux 1997; C. aspilus Moënne-Locc. 1997; C. collybioides Reumaux 1997; C. conicoides Bidaud 1997; C. herculinus Reumaux 1997; C. hinnuleoscitus Ramm \& Rob. Henry 1995; C. hinnuleus f. parincisus Bidaud, Moënne-Locc. \& Reumaux 1997; C. lepidus Moënne-Locc. 1997; C. ochraceoplicatus Reumaux 1997; C. pseudohinnuleus Bidaud, Moënne-Locc. \& Reumaux 1997; C. salicum Reumaux 1997

C. cinereoviolascens Moënne-Locc. \& Reumaux 1988; C. malachius $\mathrm{f}$. cholagogus Bidaud, Moënne-Locc. \& Reumaux 2002; C. malachius f. crinitus Bidaud \& Reumaux 2002; C. ochraceus Peck 1872

C. micro-ornatus Chevassut \& Rob. Henry 1978; C. summomaculatus Rob. Henry 1995

C. incisopunctatus Reumaux 2012

C. adalbertii var. turritus M.M. Moser 1980; C. inolens (H. Lindstr.) Bidaud 2010; C. striaepilus J. Favre 1948

C. pseudorusticus Bidaud 2002
C. lacorum A.H. Sm. 1934

C. saniosus var. paludophilus Carteret \& Reumaux 2012

C. ferrugineifolius M.M. Moser 1993; C. paraphaeochrous M.M. Moser 1993; C. subrigidipes M.M. Moser 1993 
Table 1 (continued)

\author{
Current name \\ C. parhonestus Reumaux 2012 \\ C. pearsonii P.D. Orton 1958 \\ C. pelargoniostriatulus Bidaud \& Fillion 2010 \\ C. phaeochrous J. Favre 1955 \\ C. phaeosmus Rob. Henry 1981 \\ C. pholideus (Lilj.) Fr. 1838 \\ C. piceidisjungendus Kytöv., Liimat., Niskanen \& Ammirati 2014 \\ C. pilatii Svrček 1968
}

C. pinigaudis Niskanen, Kytöv. \& Liimat. 2011

C. pinosquamulosus Kytöv., Niskanen \& Liimat. 2020

C. plumulosus Rob. Henry 1977

C. politus Niskanen, Liimat. \& Ammirati 2013

C. praepallens Peck 1887

C. praestigiosus (Fr.) M.M. Moser 1965

C. privignatus Soop 2010

C. privignipallens Kytöv., Niskanen \& Liimat. 2014

C. psammocola Kytöv., Niskanen \& Liimat. 2017

C. pseudobiformis Bidaud \& Carteret 2012

C. pseudobovinus M.M. Moser \& Ammirati 1995

C. pseudobulbosus Carteret \& Reumaux 2010

C. pseudofallax Carteret 2004

C. pseudoflabellus Bidaud 2010

C. pseudofusisporus Bidaud 2010

C. pseudophlegma Rob. Henry 1981

C. puellaris Brandrud, Bendiksen \& Dima 2015

C. punctatiformis Carteret 2012

C. quarciticus H. Lindstr. 1994

C. quercoconicus Liimat., Kytöv. \& Niskanen 2017

C. radicosissimus Moënne-Locc. 1997

C. raphanoides (Pers.) Fr. 1838

C. repertus A. Favre \& Vialard 2004

C. rigidipes M.M. Moser 1967

C. roseivelatus Kytöv., Liimat. \& Niskanen 2014

C. roseoarmillatus Niskanen, Kytöv. \& Liimat. 2011

C. roseobasilis Ammirati, Beug, Liimat., Niskanen \& O. Ceska 2016

C. roseobrunneus Carteret 2000

C. roseocastaneus Niskanen, Liimat. \& Kytöv. 2014

C. roseomyceliosus Bidaud 2009

C. roseonudipes Rob. Henry \& Moënne-Locc. 1997

C. rossicioenochelis Liimat., Kytöv. \& Niskanen 2017

C. rubipes Kauffman 1909
Younger synonyms

C. cremeolaniger P.D. Orton 1983; C. lanigeroides P.D. Orton 1983

C. pholideoides Bidaud \& Reumaux 2005; C. subpenicillatus Carteret \& Reumaux 2005; C. subpholideus Rob. Henry 1992

C. altipes Bidaud 2010; C. conocyboides Carteret 2004; C. distinctus Carteret 2012; C. pelargoniobtusus Rob. Henry 1985; C. pseudorigidus Bidaud, Carteret \& Reumaux 2012; C. udolivascens var. lilacinostipitatus Carteret 2004

C. fulguritans Reumaux 2000; C. magus Moënne-Locc. 2001; C. poirieri Reumaux 1988; C. rufescentipes Bidaud 2001; C. sensibilis Bidaud 2010

C. flavoperonatus Bidaud \& Reumaux 2012

C. propinquus Eyssart. \& Bidaud 2002; C. violaceostriatus MoënneLocc. \& Reumaux 2002

C. speciosior Bidaud, Moënne-Locc. \& Reumaux 1997; C. squamulifer Bidaud \& Reumaux 1997; C. subhelvolus Moënne-Locc. \& Reumaux 1997

C. buxiolens Bidaud 1997; C. carcharias Bidaud 1997; C. hinnuleovelatus Reumaux 1997; C. subfilamentosus Reumaux 1997 
Table 1 (continued)

Current name
C. rubricosus (Fr.) Fr. 1838
C. rubrocinctus Reumaux 1995
C. rubrovioleipes Bendiksen \& K. Bendiksen 1991
C. rumoribrunsii Bojantchev, Ammirati, Niskanen, \& Liimat. 2017
C. russulaespermus Carteret 2004
C. rusticellus J. Favre 1955
C. rusticus P. Karst. 1882
C. sagacitas Kytöv., Niskanen \& Liimat. 2020
C. sagacito-occidentalis Liimat., Niskanen, Kytöv. \& Ammirati 2020
C. saniosus (Fr.) Fr. 1838

C. saturninus (Fr.) Fr. 1838

C. scaurotraganoides Rob. Henry 1986

C. scotoides J. Favre 1955

C. sejunctifolius Rob. Henry 1995

C. semiodoratus Rob. Henry 1993

C. semivelatus Rob. Henry 1970

C. semivestitus M.M. Moser 1968

C. serratissimus M.M. Moser 1968

C. sociatus Rob. Henry 1983

C. sordescens Rob. Henry 1944

C. sordidemaculatus Rob. Henry 1981

C. sphagnoravus Liimat., Kytöv., Niskanen \& Ammirati 2017

C. spisnii Consiglio, D. Antonini \& M. Antonini 2004

C. squalidus A.H. Sm. 1942

C. stipitemirus Rob. Henry 1995

C. stuntzii S.A. Rehner \& Ammirati 1989

C. subargyronotus Niskanen, Liimat. \& Kytöv. 2014

C. subbalaustinus Rob. Henry 1991

C. subbrunneoideus Kytöv., Liimat. \& Niskanen 2014

C. subbulliardioides Rob. Henry 1970

C. subcagei Niskanen \& Liimat. 2017

C. subcarabus Liimat., Kytöv. \& Niskanen 2017

C. subcarneinatus Niskanen, Kytöv. \& Liimat. 2017

C. subcastaneus Bidaud \& Reumaux 2000

C. subcoronatus Bidaud 2001

C. suberi Soop 1990

C. subexitiosus Liimat., Niskanen, Kytöv. \& Ammirati 2014

C. subfillionii Kytöv., Niskanen \& Liimat. 2017

C. subglandicolor Niskanen, Liimat. \& Kytöv. 2017
Younger synonyms

C. calcareophilus Bidaud 1997; C. crassogriseascens A. Favre 2009; C. phaeomaculatus Rob. Henry 1989; C. safranopes Rob. Henry 1938; C. safranopes var. bulbosus Rob. Henry 1997; C. safranopes var. laevipes Reumaux 1997

C. uraceoarmillatus Bidaud 2012

C. boulderensis var. pallidulus J. Favre 1960

C. striatulorufus Moënne-Locc. 2004

C. canabarba M.M. Moser 1966; C. umidicola f. coeruleus M.M. Moser \& Ammirati 1995

C. bavaricus M.M. Moser 1983; C. luteolateritius (Velen.) G. Garnier 1991; C. rufoanuliferus M.M. Moser \& McKnight 1987; C. subaurantiomarginatus Bidaud \& Ferville 2012

C. castaneoruber Bidaud \& Reumaux 2012; C. sublucorum Carteret 2012

C. raphanicus Bidaud \& Moënne-Locc. 2008

C. griseascens Bidaud, Moënne-Locc. \& Reumaux 1997; C. immaculatus Bidaud 1997; C. nauseosmus Bidaud, Moënne-Locc. \& Reumaux 1997; C. subulatus Bidaud, Moënne-Locc. \& Reumaux 1997; C. solidus Bidaud, Moënne-Locc. \& Reumaux 1997

C. sefendens Rob. Henry 1983

C. fusisporus var. olivaceodepressus Reumaux 2010

C. terribilis Reumaux 2002

C. sordescens var. vestitissimus Eyssart. 2002; C. strenuipes var. subacuminatus Reumaux 2002

C. badioflammeus Bidaud 2008; C. laceratomarginatus Carteret \& Reumaux 2008

C. castaneus var. nigrescens Reumaux 1989; C. tenebrosus Reumaux 2001

C. urdaibaiensis Fernández Sas. 2003

C. brunneogriseus Soop 1993 
Table 1 (continued)

Current name

C. subheterocyclus Liimat., Niskanen \& Kytöv. 2017

C. subionophyllus Niskanen, Liimat. \& Kytöv. 2017

C. submilvinus Bidaud 2010

C. subminiatopus Kytöv., Niskanen \& Liimat. 2017

C. subobtusobrunneus Bidaud 2004

C. subobtusus Kauffman \& A.H. Sm. 1933

C. suboenochelis Kytöv., Liimat. \& Niskanen 2011

C. subpaleaceus Kytöv., Niskanen \& Liimat. 2017

C. subparvannulatus Moënne-Locc. \& Fillion 2010

C. subpulchrifolius Kauffman 1918

C. subrigens Kauffman 1918

C. subrimosus A.H. Sm. \& Hesler 1944

C. subscotoides Niskanen \& Liimat. 2019

C. subsedens Rob. Henry 1956

C. subserratissimus Kytöv., Liimat. \& Niskanen 2014

C. substriatus Kauffman 1932

C. subtabularis Kauffman 1918

C. subtilior J. Favre 1955

C. suillonigrescens Reumaux 2002

C. tatrensis R. Fellner \& Landa 1993

C. tenebricus J. Favre 1955

C. tigrinipes Bergeron 1997

C. tortuosus (Fr.) Fr. 1838

C. torvoides Rob. Henry 2000

C. torvus (Fr.) Fr. 1838

C. tragano-odorus Niskanen, Liimat. \& Ammirati 2020

C. traganus (Fr.) Fr. 1838

C. triangulus Rob. Henry 1983

C. truckeensis Bojantchev 2013

C. tuolumnensis Bojantchev 2013

C. turgidipes Bidaud \& Carteret 2008

C. turgidulus Bidaud 2002

C. turgidus Fr. 1838

C. ultimiionophyllus Kytöv., Niskanen \& Liimat. 2017

C. umbilicatus P. Karst. 1893

C. umbrinobellus Liimat., Niskanen \& Kytöv. 2014

C. umbrinolens P.D. Orton 1980

C. umbrinolutescens Reumaux 2004

C. uraceisporus Niskanen, Kytöv. \& Liimat. 2014

C. uraceomajalis Dima, Liimat., Niskanen \& Bojantchev 2014

C. uraceonemoralis Niskanen, Liimat., Dima, Kytöv., Bojantchev \&

H. Lindstr. 2014

C. uraceus Fr. 1838

C. urbicus (Fr.) Fr. 1838
Younger synonyms

C. miniatopus var. konradii M.M. Moser 1965

C. subcompar Bohus 1979

C. fumosifolius A.H. Sm. 1942

C. parinsignis Moënne-Locc. \& Carteret 2001; C. percavus J. Favre 1955

C. implexobrunnescens A. Favre 2009

C. bidiscendus Rob. Henry 1985; C. subamethysteus Rob. Henry 2000; C. testaceofractus Carteret \& Reumaux 2000; C. torvovelatus Reumaux 2000

C. pulchrifolius var. odorifer Hesler 1944

C. pyriodorus Kauffman 1932; C. traganus f. ochraceus M.M. Moser, Ammirati \& M.T. Seidl 1995

C. albolilascens Rob. Henry 1988; C. cuteclarus Bidaud, Moënne-Locc. \& Reumaux 2008; C. isabellae Rob. Henry 1981; C. ornithopus Rob. Henry 1970; C. productus Chevassut \& Rob. Henry 1988; C. subadelphus Rob. Henry 1981

C. cacaodiscus Liimat., Niskanen \& Kytöv. 2014

C. sericeofibrillosus Bidaud \& Boutev. 2001
Hydrocybe praecox Velen. 1939

C. alsomatii Rob. Henry 1992 
Table 1 (continued)

\begin{tabular}{|c|c|}
\hline Current name & Younger synonyms \\
\hline \multicolumn{2}{|c|}{ C. wahkiacus Ammirati, Beug, Liimat. \& Niskanen 2016} \\
\hline C. valgus Fr. 1838 & $\begin{array}{l}\text { C. depexus var. luminosus Carteret 2005; C. fuliginosus P.D. Orton } \\
\text { 1964; C. olivaceostipitatus Carteret 2012; C. orbiculozonarius Rob. } \\
\text { Henry 1983; C. rheubarbarinus Rob. Henry } 1956\end{array}$ \\
\hline \multicolumn{2}{|l|}{ C. venustissimus Bidaud 2002} \\
\hline C. venustus P. Karst. 1881 & C. traganulus P.D. Orton 1983 \\
\hline \multicolumn{2}{|c|}{ C. vernalishastensis Bojantchev, Ammirati, Niskanen, \& Liimat. 2017} \\
\hline \multicolumn{2}{|c|}{$\begin{array}{l}\text { C. vernalisierraensis Bojantchev, Ammirati, Niskanen, \& Liimat. } \\
2017\end{array}$} \\
\hline C. vernus H. Lindstr. \& Melot 1994 & C. erythrinus var. russulisporus Bohus 1979 \\
\hline \multicolumn{2}{|c|}{$\begin{array}{l}\text { C. vinaceobrunneus Ammirati, Beug, Liimat., Niskanen \& O. Ceska } \\
2016\end{array}$} \\
\hline \multicolumn{2}{|c|}{ C. vinaceogrisescens Ammirati, Beug, Liimat. \& Niskanen 2016} \\
\hline \multicolumn{2}{|l|}{ C. violaceopapillatus Bidaud 2010} \\
\hline C. vulpicolor M.M. Moser \& McKnight 1987 & C. glandicolor var. exilis J. Favre 1955; C. plicatus Bidaud 2010 \\
\hline
\end{tabular}

most common one at least in the Nordic countries. Therefore, we choose to follow the current Nordic concept of the species and propose the collection $\mathrm{H}$. Lindström et al. CFP 432 as the neotype of the species.

Cortinarius armeniacus (Schaeff.) Fr., Epicr. syst. mycol. (Upsaliae): 304 (1838) [1836-1838]

Basionym: Agaricus armeniacus Schaeff., Fung. bavar. palat. nasc. (Ratisbonae) 4: 35 (1774): sanctioned in Fr., Syst. mycol. 1: 234 (1821).

$=$ Cortinarius privignus (Fr.) Fr., Epicr. syst. mycol. (Upsaliae): 304 (1838) [1836-1838]

Types: Schaeff., Fung. bavar. palat. nasc. (Ratisbonae) 1-2: Tab LXXXI, 1774 (lectotypus hic designatus, IF 557455) as Agaricus armeniacus). Sweden, Ångermanland; Häggdånger sn, Torrom, in spruce forest with blueberry, 26 Sep 1988, coll. H. Lindström et al. CFP 809, F37506 (S, epitypus hic designatus, IF 557456), GenBank No. DQ117925 (ITS).

Illustration. Brandrud et al. (1989: pl. A46).

Descriptions of the species. Brandrud et al. (1989: pl. A46), Niskanen et al. (2012).

Cortinarius bibulus Quél., Compt. Rend. Assoc. Franç. Avancem. Sci. 9: 666 (1881) [1880]

Types: Quélet, Compt. Rend. Assoc. Franç. Avancem. Sci. 9: 666, Pl. VIII, fig. 7, 1881 (lectotypus hic designatus, IF 557457). Finland, Kainuu, Puolanka, Pihlajavaara S., old, mossy, mesic grass-herb spruce forest (Picea abies) with some Betula, Pinus sylvestris and Populus tremula, 240-270 m, 15 Sept 2005, coll. K. Liimatainen \& T. Niskanen 05-119, H6031525 (H, epitypus hic designatus, IF 557458, K, isoneotypus), GenBank No. MT934904 (ITS).

Illustration: Brandrud et al. (1992: pl. B25).
Descriptions of the species: Brandrud et al. (1992: pl. B25), Niskanen et al. (2012) as C. lilacinopusillus P.D. Orton.

Cortinarius bulliardii (Pers.) Fr. [as 'bulliardi'], Epicr. syst. mycol. (Upsaliae): 282 (1838) [1836-1838]

Basionym: Agaricus bulliardii Pers. [as 'bulliardi'], Observ. mycol. (Lipsiae) 2: 43 (1800) [1799]: sanctioned in Fr., Syst. mycol. 1: 221 (1821).

$=$ Cortinarius colus Fr., Epicr. syst. mycol. (Upsaliae): 308 (1838) [1836-1838]

Types: Bulliard, Herbier de la France: pl. 431 Fig. 3, 1780 (lectotypus hic designatus, IF 557459, as Agaricus araneosus). Sweden, Västergötland, Österplana sn, Österplana hed, decideous forest on calcareus soil (Corylus, Quercus, Tilia), 15 Sep 1986, coll. H. Lindström et al. CFP 499, F41127 (S, epitypus hic designatus, IF 557460), GenBank No. JX114942 (ITS).

Illustration. Brandrud et al. (1989: pl. A37).

Descriptions of the species. Brandrud et al. (1989: pl. A37), Niskanen et al. (2012).

Cortinarius colus Fr., Epicr. syst. mycol. (Upsaliae): 308 (1838) [1836-1838]

= Cortinarius bulliardii (Pers.) Fr., Epicr. syst. mycol. (Upsaliae): 282 (1838) [1836-1838]

Types: Paulet, Traité des Champignons: t. 99 spec. solita majus 1793-1835 (lectotypus hic designatus, IF 557461, as Hypophyllum colus). Sweden, Västergötland, Österplana sn, Österplana hed, decideous forest on calcareus soil (Corylus, Quercus, Tilia), 15 Sep 1986, coll. H. Lindström et al. CFP 499, F41127 (S, epitypus hic designatus, IF 557462), GenBank No. MT934978 (ITS). 
Illustration. Brandrud et al. (1989: pl. A37) as $C$. bulliardii.

Descriptions of the species. Brandrud et al. (1989: pl. A37), Niskanen et al. (2012) as C. bulliardii.

Notes-The protologue by Fries (1838) has a reference to Paulet's illustration of Hypophyllum colus that is desingnated as the lectotype of the species. It shows a fungus most similar to $C$. bulliardii although the lamellae are pale and decurrent. Fries (1838) describes the species with a pileus about $2.5-5 \mathrm{~cm}$ wide, a stipe about $3 \mathrm{~mm}$ wide and growing in Pinus forests near Uppsala. The width of the stipe is too narrow for $C$. bulliardii, the species has not been found from Uppsala and is normally associated with Quercus, Fagus and Corylus. Due to these contradictions, Brandrud et al. $(1989,1992)$ decided to apply the name to another species that occurs in pine forests which has a narrow stipe and a red orange veil, a character emphasized by Fries (1838). However, in this case the species chosen by Brandrud et al. (1989) does not fit with the type illustration or Fries's measurements of the pileus since C. colus sensu Brandrud et al. (1989) is a very small and slender species (pileus $0.5-3 \mathrm{~cm}$ wide). Our conclusion is that a species that would completely fit to Fries's description and the holotype does not exist. However, since the name has been widely used in the Nordic countries after its publication in Brandrud et al. (1989) it is better to stabilize the name rather than treat it as nomen dubium. Here, we choose to follow the type of the species and suggest an epitype for the species that will make $C$. bulliardii and $C$. colus synonyms.

Cortinarius craticius Fr., Epicr. syst. mycol. (Upsaliae): 282 (1838) [1836-1838]

Type: Finland, Satakunta, Ikaalinen, Seitseminen National Park, Multiharju strict protections area, old, mesic spruce forest (Picea abies) with some Pinus sylvestris, Betula and Populus tremula, 8 Sep 2005, coll. K. Liimatainen \& T. Niskanen 05-069, H6029911 (H, neotypus hic designatus, IF 557463; K, isoneotypus), GenBank No. MT934988 (ITS).

Illustration. Stockholm's herbarium, S0279; http://herba rium.nrm.se/specimens/S0279

Description of the species. Niskanen et al. (2012).

Cortinarius diosmus Kühner, Bull. mens. Soc. linn. Soc. Bot. Lyon 24: 39 (1955)

Type: France, Haute-Savoie, Environs de Samoëns; forêt de Bostan, sentier montant au chalet de Bostan, 6 Sep 1948, coll. R. Kühner 00110647 (G, lectotypus hic designatus, IF 557464), GenBank No. MT935017 (ITS).

Descriptions of the species. Niskanen et al. (2012) as $C$. argillaceosericeus ined.

Notes-Kühner (1955) made two collections of $C$. diosmus that are considered syntypes: 00110646 and 00110647 (G). The syntypes represent two different species that have the following younger names: $C$. diosmoides Rob. Henry and $C$. argillaceosericeus ined. Niskanen et al. (2012). Both species fit the original concept of $C$. diosmus, but we choose the specimen 110647 as the lectotype of $C$. diosmus (syn. $C$. argillaceosericeus ined.) since that represents the more common and more widespread species based on our current data.

Cortinarius flabellus (Fr.) Fr., Epicr. syst. mycol. (Upsaliae): 300 (1838) [1836-1838]

Basionym: Agaricus flabellus Fr., Syst. mycol. (Lundae) 1: 231 (1821): sanctioned in Fr., Syst. mycol. 1: 231 (1821).

Type: Sweden, Uppland, Lena sn, S of Salsta slott, in rich coniferous forest on calcarious ground, 23 Sep 1987, coll. H. Lindström et al. CFP 672, F44866 (S, neotypus hic designatus, IF 557465), GenBank No. MT935053 (ITS).

Illustration. Brandrud et al. (1998: pl. D35).

Descriptions of the species. Brandrud et al. (1998: pl. D35).

Notes-In Brandrud et al. (1998) three collections of C. flexipes var. flabellus (Fr.) H. Lindstr. \& Melot are presented. They represent two species D35 and D45/D34. The basidiomata in plate D35 fit best to Fries' description of Agaricus flabellus that has a dark olive to blackish brown pileus whereas the other species, represented by plates D45 and D34 sometimes has a red brown pileus. Therefore, we propose coll. H. Lindström et al. CFP 672 as the neotype of this species. The name of the other species is $C$. lindstroemii Niskanen, Kytöv. \& Liimat.

Cortinarius gentilis (Fr.) Fr., Epicr. syst. mycol. (Upsaliae): 297 (1838) [1836-1838]

Basionym: Agaricus gentilis Fr., Syst. mycol. (Lundae) 1: 212 (1821): sanctioned in Fr., Syst. mycol. 1: 212 (1821).

Type: Norway, Oppland, Dokka kn, Vest-Torpa, in spruce forest with blueberry (Picea, Salix), 15 Sep 1983, coll. H. Lindström et al. CFP 178, F256849 (S, neotypus hic designatus, IF 557466), GenBank No. EU266692 (ITS).

Illustration. Brandrud et al. (1992: pl. B31).

Descriptions of the species. Brandrud et al. (1992: pl. B31), Niskanen et al. (2012).

Cortinarius helvelloides (Fr.) Fr., Epicr. syst. mycol. (Upsaliae): 297 (1838) [1836-1838]

Basionym: Agaricus gentilis e helvelloides Fr., Syst. mycol. (Lundae) 1:213 (1821).

Type: Finland, Uusimaa, Espoo, Hindsby-Svartböle, under Alnus incana and Alnus glutinosa, among grasses, 17 Aug 2005, coll. anonymous, T. Niskanen 05-002, H6031432 (H, neotypus hic designatus, IF 557467; K, isoneotypus), GenBank No. MT935110 (ITS).

Illustration. Brandrud et al. (1989: pl. A17).

Descriptions of the species. Brandrud et al. (1989: pl. A17), Niskanen et al. (2012). 
Cortinarius hemitrichus (Pers.) Fr., Epicr. syst. mycol. (Upsaliae): 302 (1838) [1836-1838]

Basionym: Agaricus hemitrichus Pers., Syn. meth. fung. (Göttingen) 2: 296 (1801): sanctioned in Fr., Syst. mycol. 1: 230 (1821).

Type: Sweden, Skåne, Maglehem sn, "Piraten rasten", in birch forest, 21 Sep 1987, coll. H. Lindström et al. CFP 662, F44875 (S, neotypus hic designatus, IF 557468), GenBank No. MT935113 (ITS).

Illustration. Brandrud et al. (1989: pl. A31).

Descriptions of the species. Brandrud et al. (1989: pl. A31), Niskanen et al. (2012).

Cortinarius hinnuleus Fr., Epicr. syst. mycol. (Upsaliae): 296 (1838) [1836-1838]

Types: Sowerby, Col. Fig. Engl. Fungi Mushr.1: tab. 173, 1805 (lectotypus hic designatus, IF 557469). Sweden, Medelpad, Torp sn, Hussborg, in cultivated grassland under Betula, 28 Sep 1985, coll. H. Lindström et al. CFP 332, F37503 (S, epitypus hic designatus, IF 557470), GenBank No. DQ117926 (ITS).

Illustration. Brandrud et al. (1989: pl. A19).

Descriptions of the species. Brandrud et al. (1989: pl. A19).

Notes-The name $C$. hinnuleus has been collectively used for several deciduous forest species that have a yellowish brown to reddish brown pileus, distant lamellae with an earthy odour, white universal veil and strongly verrucose, subglobose to obovoidly subglobose spores. They collectively more or less fit to the Fries's protologue (Fries 1838) that describes a species with fulvous cinnamon pileus, distant lamellae and a white veil ring on the stipe that grows early in the season in deciduous forests. The species in the photograph of Brandrud et al. (1989), plate A19, fits Fries's protologue as well as Sowerby's colour plate and therefore we propose it as an epitype of the species.

Cortinarius laniger Fr., Epicr. syst. mycol. (Upsaliae): 292 (1838) [1836-1838]

Type: Finland, Joutsa, Koivuranta, W of Rakkolanselkä, fairly young, mesic to damp, spruce-dominated (Picea abies) forest with some Betula and Pinus, 30 Aug 2005, coll. K. Liimatainen, S. Miettinen \& T. Niskanen 05-019, 6029897 $(\mathrm{H}$, neotypus hic designates, IF 557471; K, isoneotypus), GenBank No. MT935187 (ITS).

Illustrations: Brandrud et al. (1994: pl. C53, mixed collection), Fries (1867-1884: pl. 156)

Descriptions of the species: Brandrud et al. (1994: pl. C53, mixed collection), Niskanen et al. (2012).

Notes - The plate C53 of $C$. laniger in Brandrud et al. (1994) is a mixed collection also including C. distortus Kauffman and therefore another specimen is proposed as a neotype here.
Cortinarius melleopallens (Fr.) Britzelm., Bot. Zbl. 51(23): 38 (1892)

Basionym: Cortinarius triformis var. melleopallens Fr., Epicr. syst. mycol. (Upsaliae): 299 (1838) [1836-1838].

Type: Sweden, Härjedalen, Storsjö sn, Flatruet, in subalpine zone with Betula, Pinus, Picea, 16 Aug 1986, coll. H. Lindström et al. CFP 433, F44880 (S, neotypus hic designatus, IF 557472), GenBank No. MT935221 (ITS).

Illustration: Brandrud et al. (1992: pl. B12)

Descriptions of the species: Brandrud et al. (1992), Niskanen et al. (2012).

Notes-Fries' protologue does not perfectly fit to any currently known Cortinarius species. Since there is no clear solution, we decide to follow the Nordic concept of this name (Brandrud et al. 1992, Niskanen et al. 2012). For more nomenclatural discussion of this name and the reasoning for the current interpretation see the booklet of Brandrud et al. (1992).

Cortinarius miniatopus J.E. Lange, Fl. Agaric. Danic. 5 (Taxon. Consp.): III (1940)

Types: Lange, Fl. Agaric. Danic. 5(Taxon. Consp.): III, Plate 103 Fig. B, 1940 (lectotypus hic designatus, IF 557473). Finland, Kainuu, Suomussalmi, Näljänkä, Lohivaara, W of Kiviaro, SW side of the forest road, NE sloping spruce forest with fairly rich grass-herb depressions, Pinus, Betula, Populus tremula and Salix spp., 230 m, 13 Sep 1997, coll. I. Kytövuori 97-1369, H6041343 (H, epitypus hic designatus, IF 557474), GenBank No. MT935228 (ITS).

Notes-This species has recently been called C. colus (see also C. colus above) in the Nordic literature and listed as a synonym of C. miniatopus in Brandrud et al. (1989). However, the concept included two species, one with large spores currently named C. subminiatopus Kytöv., Niskanen \& Liimat., (photograph Brandrud et al. (1989; A55)) and a sister species with smaller spores (7.0-9.0 x 4.5-5.5 $\mu \mathrm{m}$, av. $=7.5-8.2 \times 5.0-5.2 \mu \mathrm{m}, \mathrm{Q}=1.45-1.70$, Qav.=1.52-1.62). The macroscopic description of $C$. miniatopus by Lange (1940) fits both species well but the spore size given is $6.5-7 \times 4.3-4.5 \mu \mathrm{m}$. Although the spore size in the protologue is even smaller than that of the small-spored species we conclude that the small-spored species fits best to the original description and here propose collection H6041343 as the epitype of the species.

Cortinarius paleaceus Fr., Epicr. syst. mycol. (Upsaliae): 302 (1838) [1836-1838]

current name Cortinarius hemitrichus (Pers.) Fr., Epicr. syst. mycol. (Upsaliae): 302 (1838) [1836-1838]

Type: Sweden, Skåne, Maglehem sn, "Piraten rasten", in birch forest, 21 Sep 1987, coll. H. Lindström et al. CFP 662, 
F44875 (S, neotypus hic designatus, IF 557475), GenBank No. MT935265 (ITS).

Illustrations: Brandrud et al. (1989: pl. A31), Fries (1867-1884: pl. 160)

Descriptions of the species: Brandrud et al. (1989: pl. A31) as $C$. hemitrichus, Niskanen et al. (2012) as $C$. hemitrichus.

Notes-The name $C$. paleaceus has often been applied to C. flexipes (Pers.) Fr. coll. However, no odour, which is very typical of species of $C$. sect. Flexipedes Kytöv., Niskanen \& Liimat., is mentioned in Fries's protologue (Fries 1838) and the lamellae are described as whitish when young. In addition, a plate from Fries (1867-1884) illustrates a species with pale lamellae and context of the stipe, a species that looks like $C$. hemitrichus, and not like $C$. flexipes and relatives that have darker lamellae and stipe context. Based on this we conclude that our current interpretation of $C$. hemitrichus best represents also this species and a neotype making these two names synonyms is suggested. Both names, C. paleaceus and C. hemitrichus, were described by Fries (1838) in the Epicrisis. Here we choose to continue the use of the name $C$. hemitrichus as the current name of the species to avoid a name change and confusion.

Cortinarius pholideus (Lilj.) Fr., Epicr. syst. mycol. (Upsaliae): 282 (1838) [1836-1838]

Basionym: Agaricus pholideus Lilj., Utkast. Sv. Fl., Edn 3: 645 (1816).

Type: Sweden, Ångermanland, Säbrå sn, Näs, in dry coniferous forest with blueberry and lichen (Betula, Picea), 29 Aug 1987, coll. H. Lindström et al. CFP 602, F248484 (S, neotypus hic designatus, IF 557476), GenBank No. MT935303 (ITS).

Illustration: Brandrud et al. (1992: pl. B37).

Descriptions of the species: Brandrud et al. (1992: pl. B37), Niskanen et al. (2012).

Cortinarius praestigiosus (Fr.) M.M. Moser, Schweiz. Z. Pilzk. 43(8): 131 (1965)

Basionym: Cortinarius paragaudis var. praestigiosus Fr., Hymenomyc. eur. (Upsaliae): 379 (1874)

Type: Finland, Uusimaa, Vantaa, Tammisto, Tammisto Nature Reserve Area, herb-rich mesic to dryish mixed forest (Quercus, Corylus, Betula, Pinus sylvestris and Populus tremula), 17 Sept 2012, coll. K. Liimatainen \& T. Niskanen 12-028, H6083157 (H, neotypus hic designatus, IF 557477;

$\mathrm{K}$, isoneotypus), GenBank No. MT935314 (ITS).

Illustration: Brandrud et al. (2012: pl. E04).

Descriptions of the species: Brandrud et al. (2012: pl. E04), Niskanen et al. (2012).

Cortinarius psammocephalus (Bull.) Fr., Epicr. syst. mycol.: 301 (1838) nomen dubium
Basionym: Agaricus psammocephalus Bull., Herb. Fr. (Paris) 13: 12, tab. 531, fig. 2 (1793).

Notes - This species was described by Bulliard (1793) and the only original material is the painted figure that has been chosen as a lectotype of the species in Brandrud et al. (1998). The plate illustrates a rather slender, brown species with a wide, convex to low convex, sometimes low umbonate, scaly pileus, and a scaly stipe, the lamellae are brown. However, it is not obvious that Agaricus psammocephalus would be a Cortinarius. The illustrated basidiomata are also reminiscent of species in the genus Inocybe and the clustered growing habit reminds one of a saprotrophic fungus. The epithet psammocephalus was combined in the genus Cortinarius by Fries (1838), who intepreted it as a species growing in coniferous forests. Because Bulliard worked in the Paris region, already Brandrud et al. (1998) concluded, that Fries's species most likely is different from Bulliard's species that supposedly was growing in a deciduous forest. Currently, the name is applied to a species pair $C$. castaneopallidus Carteret/C. quercoconicus Liimat., Kytöv. \& Niskanen that usually have a much narrower, acutely umbonate pileus (Bidaud et al. 2004, plate 481; Brandrud et al. 1998, plate D57).

Taking into consideration that i) the basidiomata illustrated in the Bulliard's plate do not fit the species for which the name has currently been used, ii) the plate may represent a species from another genus, and iii) we have not found another candidate for the name from the genus Cortinarius, we refrain to use the name for a species in genus Cortinarius and treat is as a nomen dubium.

Cortinarius torvus (Fr.) Fr., Epicr. syst. mycol. (Upsaliae): 293 (1838) [1836-1838]

Basionym: Agaricus torvus Fr., Observ. mycol. (Havniae) 2: 80 (1818): sanctioned in Fr., Syst. mycol. 1: 211 (1821).

Types: Bulliard, Herb. Fr. (Paris) 2: Tab. 96, pl. 600, 1782 [1781-82] (lectotypus hic designatus, IF 557478, as Agaricus araneosus). Sweden, Skåne, Degeberga sn, Forsakar, in beech forest on calcareous ground, 17 Sep 1988, coll. H. Lindström et al. CFP 778, F248482 (S, epitypus hic designatus, IF 557479), GenBank No. MT935556 (ITS).

Illustration. Brandrud et al. (1992: pl. B13).

Descriptions of the species. Brandrud et al. (1992: pl. B13), Niskanen et al. (2012).

Cortinarius traganus (Fr.) Fr., Epicr. syst. mycol. (Upsaliae): 281 (1838) [1836-1838]

Basionym: Agaricus traganus Fr., Observ. mycol. (Havniae) 2: 82 (1818): sanctioned in Fr., Syst. mycol. 1: 217 (1821).

Types: Schaeff., Fung. bavar. palat. nasc. (Ratisbonae) 1-2: Tab 56, Fig. I-V, 1774 (lectotypus hic designatus, IF 557480), as Agaricus amethystinus). Sweden, 
Ångermanland, Härnösand, Härnön at Myran, in dry sandy pine forest, 13 Sep 1988, H. Lindström et al. CFP763, F248486 (S epitypus hic designatus, IF 557481), GenBank No. MT935361 (ITS).

Illustration. Brandrud et al. (1994: pl. C04).

Descriptions of the species. Brandrud et al. (1994: pl. C04), Niskanen et al. (2012).

Notes-The protologue by Fries (1818) is very short but mentions the main characteristics of the species currently considered as C. traganus (Brandrud et al. 1994; Niskanen et al. 2012): Basidiomata with a smell. Pileus pale lilac, stipe whitish purplish and bulbous, context yellow. Fries (1818) also refers to an illustration of Schaeffer (1774) that then becomes the type of the species. A majority of the figures in the illustration represent our interpretation of $C$. traganus (Fig. I-V), but Fig. VII clearly shows a typical characteristic of $C$. cyanites Fr.: the context of the stipe and pileipellis have become vinaceous red on exposure. In Fig. IX the spores are round which does not fit either of the above species, a potential species could be found from $C$. sect. Anomali where species with round spores and bluish colours occur. It seems that the type of $C$. traganus is a mixed illustration, but since the majority of the figures and the protologue fit the current concept of $C$. traganus, we here choose an epitype to support this interpretation.

Cortinarius turgidus Fr., Epicr. syst. mycol. (Upsaliae): 278 (1838) [1836-1838]

Types: Battarra, Fungorum agri Arimensis historia: tab. 9 fig. C, 1755 (lectotypus hic designatus, IF 557482; as Monomyces ventricosus). Sweden, Bohuslän, Sotenäs, Tossene, E of Bovallstrand, Hogsäms bokskog, Fagus forest with some Betula and Populus, seashells on ground, 29 Sep 2004, coll. K. Liimatainen \& T. Niskanen 04-1020, H7017832 (H, epitypus hic designatus, F 557483; K, isoepitypus), GenBank No. MT935565 (ITS).

Illustration: Brandrud et al. (1992: pl. B58).

Descriptions of the species: Brandrud et al. (1992: pl. B58), Niskanen et al. (2012).

\section{New combinations}

Cortinarius colorius (Bidaud) Niskanen, Dima \& Liimat. comb. nov.

IF 557484

Basionym: Cortinarius ignifluus var. colorius Bidaud, in Bidaud et al., Atlas des Cortinaires (Meyzieu) 6: 190 (1994)

Cortinarius iners (Bidaud) Liimat., Dima \& Niskanen comb. nov.

IF 557485
Basionym: Cortinarius duracinus f. iners Bidaud, in Bidaud et al., Atlas des Cortinaires (Meyzieu) 17(1): 1176 (2008)

\section{Discussion}

\section{Studies of type specimens}

There are two ways for naming a barcode in a sequence database: either sequence a named voucher specimen based on a morphological identification or sequence a type specimen. Paradoxically, the first approach is currently the most widely used although the core reason for using the DNAbased identification is the unreliability of the morphological identification. The gold standard should be sequencing the type specimens to achieve an unambiguous, good quality identification database, but this unfortunately has thus far been generally neglected.

To improve the sequence-based identification of the important ectomycorrhizal genus Cortinarius and create a solid base for future taxonomic work 482 type specimens were sequenced. This is more than twice as many as the largest type study of Cortinarius so far (Liimatainen et al. 2014). We were able to successfully sequence many old type specimens; 105 types which were over 50 years old and 18 over 100 years old. This shows that most available Cortinarius type specimens can likely be sequenced regardless of the age of the specimen. The dataset, including the already published type sequences in this group, contains a total of 363 species. About half of these species' names, altogether 184, are published now for the first time in GenBank, thus doubling the reliably of barcoded species of Cortinarius, subgen. Telamonia in the public sequence databases. Also $33 \%$ of the species represented here have been described over the last decade using DNA sequences alongside morphology and ecological data. Adding DNA tools for fungal taxonomy has accelerated the process of discovering and describing fungus diversity.

\section{Synonyms}

Our dataset shows that many species have been described several times. Of the 363 species recognized in this study $31 \%$ have a synonym, the synonym rate is even higher with species described using only morphological characters $(46 \%)$. The two main reasons for synonyms are that the interpretation of the existing names has been challenging and there have been problems based on the morphological species concept. The high number of species, convergent evolution and the small number of useful morphological characters for classification have not made the task any easier. Also, the lack of uniform and stable 
infrageneric classification has made it more difficult to find potential, already existing descriptions of the species and thus many species have been subsequently named as new again. In the future, the problem of synonyms will be much reduced when sequences from type specimens are available and the description of new species without ITS barcodes are strongly discouraged.

One example of the difficulty of interpret existing names is C. impolitus Kauffman. It was the species described the most times by multiple authors over decades, e.g. by Kauffman (1918) and Smith (1944) from North America and by Velenovský (1939), Pearson (1946), Favre (1955) and Lindström (Brandrud et al. 1998) from Europe (Table 1 and Figure 1). The species is small and brown which partly explains the problem but it also has two good characters, odour of Pelargonium in the lamellae and narrow basidiospores, but despite these characteristics it has been very challenging to recognize it from the works of different mycologists based on morphology only.

Examples of the second problem, the challenges of using the morphological species concept, are C. macropodius Rob. Henry and C. luridus Rob. Henry that overall had the highest number of synonyms, 13 and 9 respectively. In this case, all synonyms come from the French authors and are due to a too narrow species concept. Some of the synonyms are also placed in different infrageneric groups in their classification system. This error rate and unnatural classification make it very difficult to use the earlier parts of the Atlas des Cortinaires series for identification of Cortinarius. However, the individual descriptions of the species are usually of good quality and 61 species names that have been described by the team are the oldest names for the species: representing about $15 \%$ of all the currently known species of $C$. subgenus Telamonia. In recent years they have also included molecular data into their work which has greatly improved the outcome (e.g. Bidaud et al. 2017).

When looking at the rate at which the different authors described synonyms it is self-evident that it was easier to describe new species earlier when more species were undescribed. For example, the error rate of Kauffman is only 7\% whereas Smith's error is double that, most likely because he was partly describing the species from the same area where Peck and Kauffman had previously worked. Half of the Smith's synonyms are Kauffman's species. The error rate of Moser and Henry are rather similar, which is a bit surprising since they mainly worked in different habitats and with a different species concept.

\section{Interpretation and typification of the early names without type materials}

Many early names without type specimens have been redescribed by later authors. From all the old names used in this study only 10 of them are without synonyms: $C$. armillatus (Fr.) Fr., C. bibulus, C. bovinus Fr., C. cinnabarinus Fr., C. colymbadinus Fr., C. dolabratus Fr., C. evernius (Fr.) Fr., C. gentilis, C. glandicolor (Fr.) Fr., and $C$. helvelloides. About half of them are rather characteristic and easy to interpret so taxonomists after Fries understood his concept and therefore did not describe those species again, i.e. C. armillatus and $C$. evernius. On the other hand, some of these species are really difficult to interpret and might not have been described again just because of the restricted distribution, infrequent occurrence or just a matter of chance, i.e. $C$. bovinus and C. dolabratus.

Interpreting the early names, like those of Fries and Persoon, when often no physical specimen is left to study and the descriptions themselves are short, vague and without microscopical characters, is extremely difficult. In many cases their species concept most likely included several species and was too generalized. They surely did their best but the state of knowledge in those times was far from what we know now. For example, $C$. paragaudis and $C$. praestigiosus, two species which based on current, widely accepted concept are far from each other both phylogenetically and morphologically, were included as varieties of one species in Fries' concept (Fries 1874). In Cortinarius sect. Bovini only one species was described by Fries although the section includes at least seven species in Sweden (Niskanen et al. 2013). Of course, Fries might not have found all those species in the areas he collected or did not have time to work with them, but it is still rather certain that many Fries' names included several species. Thus, due to the broad species concept there often is not any correct one candidate for epi- or neotypification. And even if there has been a clear concept behind the early species descriptions, it is often very difficult to interpret based on short and vague descriptions.

The interpretation of a name based only on morphology is a demanding, often impossible, task. In this study ca. $80 \%$ of the species described by Fries have been described again. The poor record can not be explained by a few poor studies or unprofessional authors-all major Telamonia authors have misinterpreted Fries' names or simply overlooked them. Studying the type specimens of Karsten's species gave a similar result. Karsten's descriptions are somewhat better than Fries' since they also include microscopical characteristics, but the critical difference is that Karsten's specimens are available and can be sequenced, thus we really can confirm the true identity of his species. The result was that all the seven Telamonia species described by Karsten, which we studied, have been redescribed later by other authors 
confirming the conclusion from Fries' materials. No current data supports the claim that the early names could be interperated correctly and consistently by anyone.

Because of the problems mentioned above the interpretation of early names in general is not a very meaningful thing to do and often the outcome is highly questionable. The majority of early names should probably be treated as nomen dubium. Therefore, we only typified those early names that have been widely used, e.g. appear on many national check lists or are commonly used in books like Funga Nordica (Niskanen et al. 2012). In these cases, the typification is a quicker and a more efficient way to stabilize nomenclature than trying to convince users to stop using the name. Also, it is important to point out that when typifying early names, we do not claim that the outcome would be correct, i.e. would represent the original concept of the author. We simply try to find the species that would best fit to the original description and in the case of several equally suitable candidates choose the most practical solution, i.e. the one that causes fewest changes in the current use of the name, the species itself would be the most common and wide spread of the candidate species and/or the easiest to recognize.

Another problem with the old names is the references to the illustrations. At those early times authors did not know that the references would later turn out to be the most important part of the descriptions-based on the current International Code of Nomenclature for algae, fungi, and plants (https://www.iapt-taxon.org/nomen/main.php), they are considered as 'original material' of the species. At the time there was not a huge amount of published illustrations to choose from. It seems that in some cases Fries referred to an illustration that did not fit perfectly to his concept of the species but was the closest one with some similarity. This is e.g. obvious with $C$. colus and $C$. turgidus.

\section{Nomenclatural coverage of the dataset and conclusions}

In this study we tried to sequence all species level type specimens belonging to Cortinarius subgen. Telamonia that have not been previously studied. Our aim also was to stabilize all commonly used early names for which a type specimen does not exist. Obviously, all names in Telamonia are not in this dataset. Some type specimens could not be sequenced, especially Hongo's and Murrill's types failed almost without exception. Also, Henry's material was difficult to sequence and in addition, many of his type specimens were not found, the names are nomenclaturally invalid, or had other problems. Most of the Peck's material could not be acquired from NYS during the time of the molecular study of this paper. Some of Favre's type specimens were too small to sample or have already been sequenced but not published by other authors. Melot's type specimens are in his personal collection and despite several attempts to aquire them on loan, they were not avalaible for molecular study. Unless this situation changes the identity of the names remains unclear and it would be better not to use them to avoid confusion rising from the different interpretations of the names. There are a few authors whose materials we have not studied, e.g. Bon and Lamoure, but the number of Telamonia species they described is relatively small, only some tens of species.

After this study there will only be a few dozen valid names that have not yet been studied with molecular methods and where the type specimens are good quality for sequencing and available for study. Most likely many of them have an earlier name which already have been studied. There are a few exceptions, however, for example if one is working with the sub-alpine Telamonia species the names described by Favre (e.g. 1955) and Lamoure $(1977,1978)$ are relevant, for Eastern North America species described by C. H. Peck's should be checked (Burnham 1919; Gilbertson 1962), and for European Mediterranean areas the works of local authors would be appropriate to study (e.g. Mahiques and Ortega 2002). Otherwise, if a new sequence does not have $\geq 99 \%$ similarity to any published type sequence it can be rather certain that it derived from an undescribed species, given that the quality of the sequence is good.

Overall, our data set contains about 300 species from Europe and 150 species from North America and many of which they have in common. There may only be a few hundred more Telamonia species to be found from Europe, but certainly in North America the quest has just begun. The situation in Africa, Asia and Central and South America is praticially unknown, but it would not be an exaggeration to predict that the world-wide diversity of Cortinarius subgen. Telamonia would be a four-digit number. Thus far, members of the subgenus have not been found in the Nothofagus forests of New Zealand (Soop et al. 2019) and from Nothofagus forests of South America only one species is confirmed (Garnica et al. 2005).

As species are discovered and named the easier the identification based on ITS will become. Unfortunately, the same does not apply to morphological identification. All the current keys we use would require extensive rewriting and even though there often are morphological and/or ecological differences between the species, identifying many of the species of Telamonia using keys without deeper experience and knowledge of the group will be challenging if not impossible. Having local keys (i.e. Scandinavian boreal Telamonias or Telamonias of the Pacific North West) and in certain cases only trying to identify sections or species complexes rather than species would be the most realistic approach when using morphological identification.

Many times, the biggest obstacle for efficient identification and naming of alfa diversity are the nomenclatoral problems, i.e. what is the correct name for the species or is 
it an undesribed one? The species of $C$. subgenus Telamonia have been considered one of the most challenging cases in the Agaricales at the species level. Its high diversity combined with convergent, similar appearing taxa have earned it a reputation of being an impossible group to study, one better left in the forest. Our study shows that nomenclatoral problems, even in difficult groups like Telamonia, can be solved and identification based on ITS barcodes becomes an easy task even for non-experts.

\section{Infrasubgeneric classification}

\section{Relationships of the sections within Cortinarius subgen. Telamonia}

The relationships of the sections within Telamonia remain unclear in our phylogenetic analysis. The grouping of the sections in the tree, however, does not seem random and makes sense when compared to the morphological characteristics. Therefore, the main findings that we feel would be of importance are summarized below and could be used as starting hypotheses for future studies.

Based on our phylogenetic analysis $C$. subgen. Telamonia is roughly divided into two main entities (Fig. 2): (i) The basal groups of the tree ("Basal Telamonias") that only contain species with medium- to large-sized basidiomata (the apex of the stipe is $>4 \mathrm{~mm}$ wide) with the exception of a few species in $C$. sect. Brunnei. (ii) The monophyletic upper part of the tree ("Crown Telamonias") that mainly contains species with small basidiomata (the apex of the stipe is < $4 \mathrm{~mm}$ wide), and the following sections including species with mainly small- to medium-, less commonly large-sized basidiomata: Hinnulei Melot, Rubricosi Moënne-Locc. \& Reumaux Leiocastanei Niskanen, Kytöv. \& Liimat., and the monotypic sections Pseudoduracini Liimat., Niskanen \& Kytöv., Friesiorum Liimat., Kytöv. \& Niskanen, and Vinaceobrunnei Ammirati, Niskanen \& Liimat.. The most basal part of this clade also includes sections Anthracini Melot, Crassispori Kytöv., Niskanen \& Liimat. and Squalidi Liimat., Ammirati \& Niskanen.

Within the "Crown Telamonias" some further grouping can be observed. Brandrud et al. (1989) initially classified the species with small basidiomata into two sections, Incrustati Melot and Hydrocybe (Fr. ex Rabenh.) P. Karst. Although not forming well supported clades, these two earlier groups seem to correlate with the phylogeny to some extent. In the Fig. 2 the groups are named as /Squamicybe (Incrustati s. Brandrud et al.) and /Erubescentes (Hydrocybe s. Brandrud et al.). The new names are introduced because the type species of sect. Hydrocybe, Cortinarius duracinus, does not belong to "Crown Telamonias" but to "Basal Telamonias" and the type species of sect. Incrustati, C. luxnymphae, was not available for study and thus the identity of the species remains unclear.

The previous members of the $C$. sect. Incrustati are all placed in/Squamicybe (Fig. 2) in two monophyletic groups/ Eusquamicybe and/Paludosi but the group also includes sections of species with medium- to large-sized basidiomata. Many species of this group have a \pm scaly pileus, a universal veil that forms distinct girdles on the stipe and a stipe/ context of the stipe that becomes darker towards the base, especially with age. No part of the basidiomata turns reddish (except in $C$. sect. Rubrocincti that resembles more the species in /Erubescentes). Typical examples of this group are $C$. flexipes $(C$. sect. Flexipedes), $C$. hemitrichus $(C$. sect. Paleacei) and C. saniosus (Fr.) Fr. (C. sect. Saniosi Moënne-Locc. \& Reumaux) and from the larger species C. hinnuleus (C. sect. Hinnulei) and C. rubricosus (Fr.) Fr. (syn. C. safranopes Rob. Henry, C. sect. Rubricosi). Species associated with Alnus, i.e. C. bibulus (C. sect. Bibuli), C. griseocarneus Carteret (C. alnetorum (Velen.) M.M. Moser sensu Brandrud et al. 1989, C. sect. Alnicolarum) and C. helvelloides ( $C$. sect. Helvelloides), also belong to this larger group. Together with $C$. sect. Saniosi they form a monophyletic group /Paludosi, although without support, indicating that within $C$. subgen. Telamonia the ability to form mycorrhizae with Alnus may only have evolved once.

A majority of the species classified earlier in $C$. sect. Hydrocybe are placed in another, monophyletic, group / Erubescentes (Fig 2). They all have small basidiomata and a smooth pileus and in most species the stipe/context of the stipe does not become darker towards the base. In addition, in quite a few species either the base of the stipe, universal veil and/or basal mycelium turns \pm reddish with time. The universal veil varies from indistinct to forming distinct girdles on the stipe. Typical examples of this group are $C$. fuscoalbus Kytöv., Niskanen \& Liimat. (C. sect. Atroalbi Niskanen, Kytöv. \& Liimat.), $C$. decipiens (Pers.) Fr. ( $C$. sect. Castanei Moënne-Locc. \& Reumaux), C. praestigiosus (C. sect. Praestigiosi Kytöv., Niskanen \& Liimat.) and $C$. vernus H. Lindstr. \& Melot (C. sect. Verni Kytöv., Niskanen \& Liimat.).

\section{Sections}

The aim of this study was not to solve the infrasubgeneric classification of $C$. subgen. Telamonia but to show the preliminary placement of the studied species and existing sections (Figs. 1 and 2). Examples of the species belonging to the sections are shown in Supplementary Fig. 1-11. We included representative photographs for all but the following 
three sections: $C$. sect. Cacaodisci Kytöv., Niskanen \& Liimat., $C$. sect. Pseudoduracini, and $C$. sect. Squalidi. In this study, 80 previously described sections and 9 subsections are used, and additional 11 section names are considered synonyms. A small number of species are not currently placed in any of the sections. The "Basal Telamonias" with mediumto large-sized basidiomata have been easier to study and are thus better known and only four species, $C$. hepaticus Kytöv., Niskanen \& Liimat., C. hillieri Rob. Henry, C. uraceisporus Niskanen, Kytöv. \& Liimat. and one $C$. sp., remain outside the currently accepted sections. In the "Crown Telamonias", that have been more overlooked mainly due to their small size, 18 species included in our phylogenetic analysis remain unclassified. Some of them, like C. denigratus Ammirati, Beug, Niskanen, Liimat. \& O. Ceska and the related $C$. spp from North America that form a monophyletic group and differ $>4 \%$ ( $>20$ indels and substitutions) from other species of Cortinarius subgen. Telamonia, might be considered as a new section in the future. Some may be grouped with existing sections with futher analysis using additional DNA regions, i.e. C. ferrugineovelatus Kytöv. Liimat. \& Niskanen and $C$. umbrinobellus Liimat., Niskanen \& Kytöv. that share morphological characteristics with the species in $C$. sect. Praestigiosi but were currently placed in a basal position of the branch containing that section.

We wanted the section names to be as unambiguous as the species names as far as possible and therefore we only accepted section names that can be interpreted without a doubt, i.e. the type specimen of the type species of a section is sequenced. Exceptions were made with four names: sect. Anthracini Melot, sect. Brunneotincti M.M. Moser, sect. Cinnabarini Melot, and sect. Parvuli Melot. For these sections we have not been able to study the type specimen of the type species for several reasons or the sequencing failed but we believe that the concept of the type species is rather uniform and clear (e.g. Niskanen et al. 2012). Therefore, it seems acceptable to use these common section names. We are aware that this kind of approach is risky as the case of sect. Testaceofolii Liimat., Niskanen \& Kytöv. shows. At the time, it was clear that $C$. biformis Fr. sensu Funga Nordica (e.g. Niskanen et al. 2012) was a different species than $C$. testaceofolius $\mathrm{H}$. Lindstr. \& Soop. However, later it turned out that the neotype Moser had selected for $C$. biformis was in fact an older synonym for $C$. testaceofolius, an outcome that no one had previously thought possible. Therefore, sect. Biformes Moënne-Locc. \& Reumaux and sect. Testaceofolii are now synonyms.

If the species concept is often difficult to apply, then classification above species becomes even more subjective. In general, we should try to avoid having too many monotypic entities since they are less meaningful in classification.
However, the risk with bigger entities is having units which would have very little, if any, exclusive morphological characters that would define those groups, since one of the main reasons of having a higher-level classification is to recognize groups with unique character states. For example, $C$. armillatus, $C$. sect. Armillati, $C$. subgen. Telamonia, genus Cortinarius represent four levels of classification in which the species $C$. armillatus belongs to all four groups that have their own, unique defining characters that other groups in higher or lower levels do not have.

In this study, one example of the difficulties of delimiting a section is $C$. sect. Uracei. With a wider concept it includes several previously recognized sections, $C$. sect. Cinnabarini, $C$. sect. Colymbadini, and $C$. sect. Miniatopodes MoënneLocc. \& Reumaux, that all form a monophyletic clade with good support value and morphological differences from $C$. sect. Uracei s. str. Therefore, keeping all above-mentioned sections separate would be an arguable choice, but then there would be at least more than four monotypic sections inside the clade Uracei that would need a new name. In this case we have currently delimited $C$. sect. Uracei in a broad sense because the group is also supported by morphological characters. The other existing sections are treated at the subsection level.

We have tried to delimit the sections to be the widest monophyletic group with a reasonable support value and with at least some shared morphological character states. This approach and level of grouping mainly corresponds to the concepts previously used to delimit the sections in the genus Cortinarius in the era of molecular data (e.g. Ammirati et al. 2013, 2017; Dima et al. 2014; Liimatainen et al. 2015, 2017, 2020; Niskanen et al. 2009, 2011, 2013; San Fabian et al. 2018; Soop et al. 2019).

The sections identified here vary from monotypic entities i.e. C. sect. Brunneocalcarii Niskanen, Liimat. \& Kytöv. to middle-sized groups i.e. $C$. sect. Armillati and $C$. sect. Disjungendi to very diverse groups i.e. $C$. sect. Bovini and $C$. sect. Uracei. The imbalance is unlikely to be solved due to the speciation history of different groups, likely some of them have diversified more than the others which has led to the current species-poor and species-rich groups. Also, this dataset only contains a fraction of the true diversity of $C$. subgen. Telamonia worldwide and therefore the number and the species diversity of sections will change when more data are available. Most of the monotypic sections will most likely turn out to be multi-species sections as shown e.g. by Soop et al. (2019).

This is the first extensive phylogenetic study of $C$. subgen. Telamonia. The great majority of sections and species are shown in a phylogenetic context for the first time. Also, many sections previously included in phylogenetic studies 
now contain more species and therefore seem to have better support values. For example, Harrower et al. (2011) used the same two DNA regions, ITS and LSU, in their study and got less than BS 50\% support for C. sect. Firmiores (Fr.) Hennings when including three species in their analysis. In our study that contains 20 species the support value for the same section was BS $88 \%$. For $C$. sect. Armillati the corresponding values were BS 54\% ( 2 species) and $90 \%$ (7 species).

\section{Conclusions}

Fig. 1 shows our current view of the number of the sections in Cortinarius subgen. Telamonia and which species we include in them. The earlier delimitations based on morphology have been partly incorrect and included only a part, often a small fraction, of the species (Bidaud et al. 2017; Brandrud et al. 2012; Niskanen et al. 2012). The classification presented here is a major step forward and can be used as a basis for a more thorough revision of morphological characterstics of the groups in the future.

Now that the nomenclatoral history of the last 100 years has been sorted out for many taxa, everyone can benefit from the outcome and continue to improve the understanding of this diverse group of species. Fortunately, all current Cortinarius taxonomists produce an ITS barcode of the type specimen of new species and upload and annotate the new sequence in GenBank. We hope that mycologists working on Cortinarius and other genera will build on the findings reported here.

Acknowledgements The help that we received from the curators of E, FLAS, G, H, IB, K, MICH, NYSM, O, OULU, PC, PRM, S and WTU was essential to this project. The following curators are warmly thanked: Bart Buyck, Xavier Carteret, Philippe Clerc, Regina KühnerWinkler, Ursula Peintner, and Patricia Rogers. We are grateful to Jesko Kleine for his invaluable help in the typification process. Mike Beug is thanked for providing the photos of C. albosericeus and C. vinaceogrisescens, Geert Schmidt-Stohn for providing the photos of C. phaeosmus and $C$. valgus, Jodi Friesen and Marty Kranabetter for providing the photo of $C$. ochropallens, Cathy Cripps for providing the photo of $C$. flavobasilis, and Andy Overall for providing the photo of C. punctatiformis (published in Overall (2017): Fungi - Mushrooms \& Toadstools of parks, gardens, heaths and woodlands).This work was supported by the Ministry of Environment, Finland (YM38/5512/2009), The Finnish Cultural Foundation, Daniel E. Stuntz Memorial Foundation (Univ. of Washington, USA), Swedish taxonomy Initiative-project (University of Gothenburg), Kone Foundation (FinBOL project) and the ELTE Institutional Excellence Program by the National Research, Development and Innovation Office of Hungary (NKFIH-1157-8/2019-DT). Lastly, we would like to thank the reviewers for their constructive comments, which helped us to improve the manuscript.

Open Access This article is licensed under a Creative Commons Attribution 4.0 International License, which permits use, sharing, adaptation, distribution and reproduction in any medium or format, as long as you give appropriate credit to the original author(s) and the source, provide a link to the Creative Commons licence, and indicate if changes were made. The images or other third party material in this article are included in the article's Creative Commons licence, unless indicated otherwise in a credit line to the material. If material is not included in the article's Creative Commons licence and your intended use is not permitted by statutory regulation or exceeds the permitted use, you will need to obtain permission directly from the copyright holder. To view a copy of this licence, visit http://creativecommons.org/licenses/by/4.0/.

\section{References}

Ammirati JF, Hughes KW, Liimatainen K, Niskanen T, Matheny PB (2013) Cortinarius hesleri from eastern North America and related species from Europe and western North America. Botany 91(2):91-98

Ammirati JF, Niskanen T, Liimatainen K, Dimitar B, Peintner U, Kuhnert-Finkernagel R, Cripps C (2017) Spring and early summer species of Cortinarius, subgenus Telamonia, section Colymbadini and /Flavobasilis, in the mountains of western North America. Mycologia 109(3):443-458

Bidaud A, Bellanger J-M, Carteret X, Reumaux P, Moënne-Loccoz P (2017) Atlas des Cortinaires. Pars XXIV, Èditions Fèdération mycologique Dauphiné-Savoie, Lomazzo, France

Bidaud A, Carteret X, Eyssartier G, Moënne-Loccoz P, Reumaux P (2004) Atlas des Cortinaires. Pars XIV, Èditions Fèdération mycologique Dauphiné-Savoie, Lomazzo, France

Bidaud A, Moënne-Loccoz P, Reumaux P (1994) Atlas des Cortinaires. Pars VI, Èditions Fèdération mycologique DauphinéSavoie, France

Bidaud A, Moënne-Loccoz P, Reumaux P, Carteret X, Eyssartier G (2008) Atlas des Cortinaires. Pars XVII, Èditions Fèdération mycologique Dauphiné-Savoie, Lomazzo, France

Borchsenius F (2009) FastGap 1.2. Department of Biosciences, Aarhus University, Denmark. Published online at http://www. aubot.dk/FastGap_home.htm

Brandrud TE, Lindström H, Marklund H, Melot J, Muskos S (1989) Cortinarius Flora Photographica. Vol. I (Swedish version). Cortinarius HB, Matfors, Sweden.

Brandrud TE, Lindström H, Marklund H, Melot J, Muskos S (1992) Cortinarius Flora Photographica. Vol. II (Swedish version).Cortinarius HB, Matfors, Sweden.

Brandrud TE, Lindström H, Marklund H, Melot J, Muskos S (1994) Cortinarius Flora Photographica. Vol. III (Swedish version).Cortinarius HB, Matfors, Sweden.

Brandrud TE, Lindström H, Marklund H, Melot J, Muskos S (1998) Cortinarius Flora Photographica. Vol. IV (Swedish version). Cortinarius HB, Matfors, Sweden.

Brandrud TE, Lindström H, Marklund H, Melot J, Muskos S (2012) Cortinarius Flora Photographica. Vol. V (Swedish version). Cortinarius HB, Matfors, Sweden.

Bulliard P (1793) Herbier de la France 13. Paris, France.

Burnham SH (1919) Charles Horton Peck. Mycologia 11(1):33-39. https://doi.org/10.1080/00275514.1919.12016772

Dima B, Liimatainen K, Niskanen T, Kytövuori I, Bojantchev D (2014) Two new species of Cortinarius, subgenus Telamonia, sections Colymbadini and Uracei, from Europe. Mycol Progress 13:867-879. https://doi.org/10.1007/s11557-014-0970-6

Favre J (1955) Les champignons supérieurs de la zone alpine du Parc National suisse. Ergebn. wiss. Unters. schweiz. NatnParks V 33:1-212

Fries EM (1818) Observationes Mycologicae 2. Bonnier, Copenhagen, Denmark

Fries EM (1836-1838) Epicrisis systematis mycologici seu synopsis Hymenomycetum. Uppsala, Sweden. 
Fries EM (1867-1884) Icones selectae Hymenomycetum nondum delineatorum. Nordstedt \& Son, Uppsala, Sweden.

Fries EM (1874) Hymenomycetum Eur. Uppsala, Sweden

Frøslev TG, Jeppesen TS, Læssoe T, Kjøller R (2007) Molecular phylogenetics and delimitation of species in Cortinarius section Calochroi (Basidiomycota, Agaricales) in Europe. Mol Phyl Evol 44:217-227

Galtier N, Gouy M, Gautier C (1996) SEAVIEW and PHYLO_WIN: two graphic tools for sequence alignment and molecular phylogeny. Bioinformatics 12:543-548

Gardes M, Bruns TD (1993) ITS primers with enhanced specifity for basidiomycetes: Application to the identification of mycorrhizae and rusts. Mol Ecol 2:113-118. https://doi.org/10.1111/j.1365294X.1993.tb00005.x

Garnica S, Schön ME, Abarenkov K, Riess K, Liimatainen K, Niskanen T, Dima B, Soop K, Frøslev TG, Jeppesen TS, Peintner U, Kuhnert R, Brandrud TE, Saar G, Oertel B, Ammirati JF (2016) Determining threshold values for barcoding fungi: Lessons from Cortinarius (Basidiomycota), a highly diverse and widespread ectomycorrhizal genus. FEMS Microbiol Ecol 92(4):fiw045. https://doi.org/10.1093/femsec/fiw045

Garnica S, Weiß M, Oertel B, Oberwinkler F (2005) A framework for a phylogenetic classification in the genus Cortinarius (Basidiomycota, Agaricales) derived from morphological and molecular data. Can J Bot 83:1457-1477

Gilbertson RL (1962) Index to species and varieties of fungi described by C.H. Peck from 1909 to 1915. Mycologia 54(5):460-465

Harrower E, Ammirati JF, Cappuccino AA, Ceska O, Kranabetter JM, Kroeger P, Lim S, Taylor T, Berbee ML (2011) Cortinarius species diversity in British Columbia and molecular phylogenetic comparison with European specimen sequences. Botany 89:799-810. https ://doi.org/10.1139/b11-065

Høiland K (1983) Cortinarius subgenus Dermocybe. Opera Botanica $71: 1-113$

Kauffman CH (1918) The Agaricaceae of Michigan. Biol. Ser 5 26:314-442

Katoh K, Kuma K, Toh H, Miyata T (2005) MAFFT version 5: improvement in accuracy of multiple sequence alignment. Nucleic Acids Res 33(2):511-518. https://doi.org/10.1093/nar/gki198

Katoh K, Standley DM (2013) MAFFT Multiple Sequence Alignment Software Version 7: improvements in performance and usability. Mol Biol Evol 30:772-780

Kirk PM, Cannon PF, Minter DW, Stalpers JA (2008) Dictionary of the fungi, 10th edn. CAB International, Wallingford, UK

Kõljalg U, Nilsson H, Abarenkov K et al (2013) Towards a unified paradigm for sequence-based identification of fungi. Mol Ecol 22:5271-5277

Kühner R (1955) Compeléments à la Flore analytique IV. Bull Mens Soc linn Soc Bot Lyon 24(2):39-54

Lamoure D (1977) Agaricales de la zone alpine. Genre Cortinarius Fr. sous-genre Telamonia (Fr.) Loud. Première partie. Trav Sci. Parc Nat Vanoise 8:115-146

Lamoure D (1978) Agaricales de la zone alpine. Genre Cortinarius Fr. sous-genre Telamonia (Fr.) Loud. Suite I. Trav Sci Parc Nat Vanoise 9:77-101

Lange JE (1940) Flora Agaricina Danica. 5. Copenhagen.

Liimatainen K, Carteret X, Dima B, Kytövuori I, Bidaud A, Reumaux P, Niskanen T, Ammirati JF, Bellanger J-M (2017) Cortinarius section Bicolores and section Saturnini (Basidiomycota, Agaricales), a morphogenetic overview of European and North American species. Persoonia 39:175-200

Liimatainen K, Niskanen T, Ammirati JF, Kytövuori I, Dima B (2015) Cortinarius, section Disjungendi, cryptic species in North America and Europe. Mycol Progress 14:1016. https://doi.org/10.1007/s1155 7-014-1016-9
Liimatainen K, Niskanen T, Dima B, Kytövuori I, Ammirati JF, Frøslev $\mathrm{T}$ (2014) The largest type study of Agaricales species to date: bringing identification and nomenclature of Phlegmacium (Cortinarius, Agaricales) into the DNA era. Persoonia 33:98-140

Liimatainen K, Niskanen T, San-Fabian B, Mujic AB, Peintner U, Dresch P, Furci G, Nouhra E, Matheny PB, Smith ME (2020) Cortinarius section Thaumasti in South American Nothofagaceae forests. Mycologia 112(2):329-341. https://doi.org/10.1080/00275 514.2019.1689763

Lindahl B, Nilsson RH, Tedersoo L, Abarenkov K, Carlsen T, Kjøller R, Kõljalg U, Pennanen T, Rosendahl S, Stenlid J (2013) Kauserud H (2013) Fungal community analysis by high-throughput sequencing of amplified markers-a user's guide. New Phytol 199:288-299. https://doi.org/10.1111/nph.12243

Maddison DR, Maddison WP (2017) Chromaseq: a Mesquite package for analyzing sequence chromatograms. Version 1.3. http://mesqu iteproject.org/packages/chromaseq

Mahiques R, Ortega A (2002) Cortinarius erythrofuscus (subgenus Telamonia, section Firmiores), a new species from Spain. Persoonia 17(4):657-660

Moser M (1983) Die Röhrlinge und Blätterpilze. In: Gams H (ed) Kleine Kryptogamenflora, Band II b/2, 5th edn. Gustav Fischer Verlag, Stuttgart, Germany

Niskanen T, Kytövuori I, Bendiksen E, Bendiksen K, Brandrud TE, Frøslev TG, Høiland K, Jeppesen TS, Liimatainen K, Lindström H (2012) Cortinarius (Pers) Gray. In: Knudsen H, Vesterholt J (eds). Funga Nordica, 2nd revised edition. Agaricoid, boletoid, clavarioid, cyphelloid and gastroid genera, Nordsvamp, Copenhagen, Denmark, pp 762-763.

Niskanen T, Kytövuori I, Liimatainen K (2009) Cortinarius sect. Brunnei (Basidiomycota, Agaricales) in North Europe. Mycol Res 113:182-206

Niskanen T, Kytövuori I, Liimatainen K (2011) Cortinarius sect. Armillati in northern Europe. Mycologia 103(5):1080-1101. https://doi. org/10.3852/10-350

Niskanen T, Kytövuori I, Liimatainen K, Lindström H (2013) Cortinarius section Bovini (Agaricales, Basidiomycota) in northern Europe, conifer associated species. Mycologia 105(4):977-993. https://doi. org/10.3852/12-320

Pearson AA (1946) New records and observations III. Trans. Br. Mycol. Soc. 29(4):191-209

Peintner U, Moncalvo J-M, Vilgalys R (2004) Towards a better understanding of the infrageneric relationships in Cortinarius (Agaricales, Basidiomycota). Mycologia 96(5):1042-1058

San-Fabian B, Niskanen T, Liimatainen K, Kooij PW, Mujic AB, Truong C, Peintner U, Dresch P, Nouhra E, Matheny PB, Smith ME (2018) New species of Cortinarius sect Austroamericani, sect. nov, from South American Nothofagaceae forests. Mycologia 110(6):11271144. https://doi.org/10.1080/00275514.2018.1515449

Schaeffer JC (1774) Fungorum qui in Bavaria et Palatinatu circa Ratisbonam nascuntur Icones 4. Regensburg

Schoch CL, Robbertse B, Robert V et al (2014) Finding needles in haystacks: linking scientific names, reference specimens and molecular data for Fungi. Database 1-21. 10.1093/database/bau061

Schoch CL, Seifert KA, Huhndorf A et al (2012) Nuclear ribosomal internal transcribed spacer (ITS) region as a universal DNA barcode marker for Fungi. Proc Natl Acad Sci USA 109:6241-6246

Simmons MP, Ochoterena H (2000) Gaps as characters in sequencebased phylogenetic analysis. Syst Biol 49:369-381. https://doi. org/10.1093/sysbio/49.2.369

Smith AH (1944) New and interesting Cortinarii from North America. Lloydia 7(3):163-235

Soop K, Dima B, Cooper JA, Park D, Oertel B (2019) A phylogenetic approach to a global supraspecific taxonomy of Cortinarius (Agaricales) with an emphasis on the southern mycota. Persoonia 42:261-290 
Stamatakis A (2014) RAxML Version 8: a tool for phylogenetic analysis and post-analysis of large phylogenies. Bioinformatics 30:1312-1313

Stensrud Ø, Orr RJS, Reier-Røberg K, Schumacher T, Høiland K (2014) Phylogenetic relationships in Cortinarius with focus on North European species. Karstenia 54:57-71

Suárez-Santiago VN, Ortega A, Peintner U, López-Flores I (2009) Study on Cortinarius subgenus Telamonia section Hydrocybe in Europe, with especial emphasis on Mediterranean taxa. Mycol Res 113:1070-1090

Thiers B (2013) Index Herbariorum: a global directory of public herbaria and associated staff. New York Botanical Garden's Virtual Herbarium. http://sweetgum.nybg.org/ih/
Velenovský J (1939) Novitates Mycologicae. Prague, Czech

White TJ, Bruns T, Lee S, Taylor J (1990) Amplification and direct sequencing of fungal ribosomal RNA genes for phylogenetics. In: Michael AJ, Gelfand DH, Sninsky JJ, White TJ (eds) PCR protocols: a guide to the methods and applications. Academic Press, New York, pp 315-322

Willis KJ (ed) (2018) State of the world's fungi 2018. Report. Royal Botanic Gardens, United Kingdom. 